\title{
Star formation in M 33: multiwavelength signatures across the disk
}

\author{
S. Verley ${ }^{1}$, E. Corbelli ${ }^{1}$, C. Giovanardi ${ }^{1}$, and L. K. Hunt ${ }^{2}$ \\ 1 Osservatorio Astrofisico di Arcetri, Largo E. Fermi 5, 50125 Firenze, Italy \\ e-mail: [simon; edvige; giova]@arcetri .astro.it \\ 2 INAF - Istituto di Radioastronomia-Sezione Firenze, Largo E. Fermi 5, 50125 Firenze, Italy \\ e-mail: hunt@arcetri.astro.it
}

Received 10 July 2008 / Accepted 27 September 2008

\begin{abstract}
Aims. We use different tracers, such as $\mathrm{H} \alpha$, ultraviolet (UV), and infrared (IR) emissions at various wavelengths, to study the dust and star formation (SF) conditions throughout the disk of M 33 .

Methods. We derive the radial distribution of dust, of the old and young stellar population using Spitzer and GALEX data, complemented by ground-based optical data and available surveys of atomic and molecular gas. We separate the contribution of discrete sources to the IR brightness from the diffuse emission.

Results. At 8 and $24 \mu \mathrm{m}$, discrete sources account for $\gtrsim 40 \%$ of the IR emission in the innermost $3 \mathrm{kpc}$, and for $\lesssim 20 \%$ further out. We find that stochastic emission from very small grains in the diffuse interstellar medium accounts for only $\sim 10 \%$ of the diffuse $24 \mu \mathrm{m}$ emission, and that dusty circumstellar shells of unresolved, evolved AGB stars (carbon stars) are a viable alternative. The $8 \mu \mathrm{m}$ profile suggests that PAH emission declines faster with radius than the dust continuum. In annular regions $0.24 \mathrm{kpc}$ wide, we find a mean extinction value for the stellar continuum $A_{\mathrm{V}} \sim 0.25 \mathrm{mag}$ with a weak dependence on radius, consistent with the shallow metallicity gradient observed. Dust opacity derived from the $160 \mu \mathrm{m}$ emission decreases instead by a factor of 10 from the center to edge of the star forming disk.

Conclusions. Using extinction corrected UV and $\mathrm{H} \alpha$ maps we find the global SF rate in M 33 over the last $100 \mathrm{Myr}$ to be $0.45 \pm 0.10 M_{\odot} \mathrm{yr}^{-1}$. Far-IR and total-IR luminosities can trace SF even though a high conversion factor is required to recover the effective rate. If carbon stars are powering the diffuse $24 \mu \mathrm{m}$ emission in M 33, this can trace star formation 1 Gyr ago and provide a more complete view of the SF history of the galaxy. Today the SF rate declines radially with a scale length of $\sim 2 \mathrm{kpc}$, longer than for the old stellar population, suggesting an inside-out growth of the disk.
\end{abstract}

Key words. galaxies: individual: M 33 - galaxies: ISM - galaxies: Local Group - galaxies: spiral

\section{Introduction}

Our knowledge of the conditions in the interstellar medium (ISM) that favour star formation (SF) is mostly based on our Galaxy and on luminous, gas-rich galaxies with high SF rates (SFRs). Thus, any proposed SF scenario is biased against different Hubble types, especially against quiescent galaxies with a low molecular-gas content. Moreover, our non panoramic view of the Milky Way, with our solar system deeply embedded in it, does not allow us to determine unequivocally important properties of our host galaxy, such as the global SFR and its variations across the galactic disk. High sensitivity and resolution in external galaxies are desirable in order to link properties integrated over the disk to the distribution of individual star-forming regions, to the ISM components and to large scale perturbations in the disk. Data from recent space missions, such as the Galaxy Evolution Explorer (GALEX) and Spitzer satellite, allow us to investigate these properties in nearby galaxies, before the next generation of telescopes will resolve star-forming sites in more distant ones.

The Local Group galaxy M 33 has a high SFR per unit area compared to M 31 and a low extinction towards SF regions, owing to its moderate gas content (Corbelli 2003) and its low inclination (54 ${ }^{\circ}, \mathrm{McC}$ Connachie et al. 2006). It is a rather quiescent blue disk galaxy, showing no sign of recent mergers or interaction. At a distance $D$ of only $840 \mathrm{kpc}$ (see Freedman et al. 1991) it allows us to examine its disk with detailed resolution and constitutes a local prototype for studying the ISM and its relation to SF in blue, low luminosity objects. Observations of the molecular, atomic and ionised gas in M 33 have been carried out (e.g. Corbelli \& Salucci 2000; Corbelli 2003; Engargiola et al. 2003; Heyer et al. 2004) together with metallicity surveys (Magrini et al. 2007a,b; Rosolowsky \& Simon 2008) of the young and old stellar populations across its disk. The galaxy has about $10 \%$ of the total gas content in a molecular form and a very shallow metallicity gradient from $0.5 \mathrm{kpc}$ to the edge of the star-forming disk.

In the first paper of this series, dedicated to the SF in M 33 (Verley et al. 2007, hereafter Paper I), we used Spitzer maps to select a large sample of infrared (IR) emitting sources in the $24 \mu \mathrm{m}$ map of this galaxy and to identify their counterparts in all IRAC wavebands and in $\mathrm{H} \alpha$. There is much spread in the correlation between the $\mathrm{H} \alpha$ and the IR fluxes of star-forming sites, similar to what has been found by Spitzer in the faint, metal deficient local dwarf NGC 6822 (Cannon et al. 2006). In selected areas of this galaxy the $\mathrm{H} \alpha$-to-IR ratios show variations as high as a factor of 10 , contrary to the tight correlation found in the bright galaxy, M 51 (Calzetti et al. 2005; Kennicutt et al. 2007). In a forthcoming paper (Corbelli et al. 2008, in preparation) we will use multiwavelength photometry to characterise how stars form in individual star-forming sites in M 33.

In this paper, the second of this series, we focus on the integrated IR emission and on its radial variations to establish the 
role of discrete star-forming regions in powering the dust emission across the disk. Indeed, there has been a long debate on the role of localised warm sources in powering the dust emission and the IR fluxes in general. Comparing IRAS and $\mathrm{H} \alpha$ observations of M33, Rice et al. (1990) and Devereux et al. (1997) found that more than $70 \%$ of the far infrared (FIR) is linked to massive star complexes. On the other hand, based on ISO observations, Hippelein et al. (2003) suggested that a substantial part of the FIR emission could be due to the cold component heated by the diffuse interstellar radiation field (ISRF). Part of the difficulties of previous surveys in determining the grain heating source had to do with the difficulties in resolving sources from IRAS and ISO observations. With Spitzer, we can now probe very faint $\mathrm{H}$ II regions and tackle this problem more effectively. In Paper I we have shown that discrete sources account for less than half of the IR emission at 8 and $24 \mu \mathrm{m}$. Understanding the origin of these high diffuse fractions in M 33 will be one of the goals of this paper. We will examine dust heating sources and the contribution of dust in different environments (diffuse ISM, H II regions, evolved stars) to the IR emission at several wavelengths as we move from the center to the outermost regions of M 33. We then use M33 as a prototype to test the reliability of UV continuum fluxes, recombination lines and IR continuum fluxes (see, e.g., Kennicutt 1998, for a complete review), as tracers of SF to possibly understand more distant galaxies, similar to M 33 but unresolved by present instruments.

The plan of the paper is the following: in Sect. 2 we present the data, in Sect. 3 the integrated luminosities in UV, $\mathrm{H} \alpha$ and IR and their radial profiles, as well as the atomic and molecular gas distributions. Optical and UV extinction and dust abundance are presented in Sect. 4. In Sect. 5 we discuss the origin of the IR emission in discrete sources and in the more diffuse component, with particular emphasis on the $24 \mu \mathrm{m}$ band. In Sect. 6 we discuss different tracers of SFRs and Sect. 7 summarises our main results.

\section{Observations and data sets}

\subsection{Ultraviolet data}

To investigate the UV continuum emission of M33, we make use of observations by the Galaxy Evolution Explorer (GALEX) mission (Martin et al. 2005). We use the data distributed by Gil de Paz et al. (2007). A description of GALEX observations of M 33 in the far-UV (FUV, 1350-1750 $⿱$ ) and near-UV (NUV, 1750-2750 A) can be found in Thilker et al. (2005), together with the relevant information on data reduction and calibration procedures.

\subsection{Ho data}

We use the narrow-band $\mathrm{H} \alpha$ image of M33 obtained by Greenawalt (1998). The reduction procedure, using standard IRAF $^{1}$ tasks to subtract the continuum emission, is described in detail in Hoopes \& Walterbos (2000). The total field of view of the image is $1.75 \times 1.75 \mathrm{deg}^{2}\left(2048 \times 2048\right.$ pixels of $\left.2{ }^{\prime \prime} 0\right)$.

\footnotetext{
${ }^{1}$ IRAF is distributed by the National Optical Astronomy Observatories, which are operated by the Association of Universities for Research in Astronomy, Inc., under cooperative agreement with the National Science Foundation.
}

\subsection{Infrared data}

The MIR and FIR data of M33 are those provided by the Spitzer InfraRed Array Camera (IRAC) and Multiband Imaging Photometer for Spitzer (MIPS) instruments (Werner et al. 2004; Fazio et al. 2004; Rieke et al. 2004). The complete set of IRAC $(3.6,4.5,5.8$, and $8.0 \mu \mathrm{m})$ and MIPS $(24,70$, and $160 \mu \mathrm{m})$ images of M33 is described in Paper I: the Mopex software (Makovoz \& Marleau 2005) was used to gather and reduce the Basic Calibrated Data (BCD). The complete field of view observed by Spitzer is very large and allows us to achieve a complete view of the star-forming disk of M33, in spite of its relatively large extension on the sky. The images were background subtracted, as explained in Paper I. The spatial resolution measured on the images are $2^{\prime \prime} 5,2^{\prime \prime} 9,33^{\prime \prime} 0,33^{\prime \prime} 0,6^{\prime \prime}, 16^{\prime \prime}$, and $40^{\prime \prime}$ for IRAC 3.6, 4.5, 5.8, 8.0 $\mu \mathrm{m}$ and MIPS 24, 70, and $160 \mu \mathrm{m}$, respectively. The absolute photometric calibration uncertainties are better than 10\% for all IRAC (Fazio et al. 2004; Reach et al. 2005) and the MIPS $24 \mu \mathrm{m}$ bands (Engelbracht et al. 2007) and are better than $20 \%$ for the MIPS 70 and $160 \mu \mathrm{m}$ channels (Rieke et al. 2004).

\subsection{1-cm and millimeter data}

The radio and $\mathrm{mm}$ databases used to infer the atomic and molecular gas distributions are: Westerbork Radio Synthesis Telescope (WRST) array (Deul \& van der Hulst 1987, 24" $\times 48^{\prime \prime}$ spatial resolution) and Arecibo single dish (Corbelli \& Schneider 1997, $4^{\prime}$ sp. res.) 21-cm line data for the atomic gas; Berkeley Illinois Maryland Association (BIMA) array (Engargiola et al. 2003, $13^{\prime \prime}$ sp. res.) and Five College Radio Astronomy Observatory (FCRAO) single dish (Corbelli 2003, 45" sp. res.) for molecular gas emission as traced by the $\mathrm{CO} J=1-0$ rotational line.

\section{Integrated emission and radial profiles}

The sudden drop in the $\mathrm{H} \alpha$ emission observed by Kennicutt (1989) in M33, and also shown later in this section, defines the extent of the star-forming disk to be $7 \mathrm{kpc}$ in radius. In this section, we first estimate the total luminosity of M 33 in UV, $\mathrm{H} \alpha$ and IR wavelengths by integrating the observed fluxes over the star-forming disk; next we extract radial profiles of elliptically averaged fluxes at IRAC and MIPS wavelengths and also from the FUV, NUV, and $\mathrm{H} \alpha$ images. We will use these profiles to evaluate the radial dependence of the average extinction which we then use to derive extinction corrected profiles and total luminosities in UV and $\mathrm{H} \alpha$. The IR emission (IRAC+MIPS) will be considered to be optically thin: luminosities are not corrected, and face-on values for the surface brightness are given by correcting only for the disk inclination to the line of sight $\left(54^{\circ}\right)$.

\subsection{Integrated luminosities}

The IR luminosities within $7 \mathrm{kpc}$ for the different IRAC and MIPS bandpasses ${ }^{2}$ are given in Table 1, together with the $3-1000 \mu \mathrm{m}$ total-infrared luminosity (TIR) derived according to the Dale \& Helou (2002) relation:

$$
F(\text { TIR })=10^{-14} \times\left(19.5 F_{v}(24)+3.3 F_{v}(70)+2.6 F_{v}(160)\right),(1)
$$

with $F$ (TIR) in $\mathrm{W} \mathrm{m}^{-2}$ and $F_{v}$ in Jy. The TIR luminosity of M 33 is $1.8 \times 10^{9} L_{\odot}$. The TIR luminosity we estimated from 8

\footnotetext{
${ }^{2}$ Following Calzetti et al. (2005), the convention $F, I, L$ (band) = $v F_{v}, v I_{v}, v L_{v}$ (band) is adopted.
} 
Table 1. Total fluxes and luminosities within $7 \mathrm{kpc}$. $\mathrm{H} \alpha$, FUV and NUV luminosities corrected for extinction are also shown.

\begin{tabular}{cccc}
\hline $\begin{array}{c}\text { Wavelength } \\
{[\mu \mathrm{m}]}\end{array}$ & $\begin{array}{c}\text { Total fluxes } \\
{[\mathrm{Jy}]}\end{array}$ & $\begin{array}{c}L \\
{\left[\mathrm{erg} \mathrm{s}^{-1}\right]}\end{array}$ & $\begin{array}{c}L \text { (corr) } \\
{\left[\mathrm{erg} \mathrm{s}^{-1}\right]}\end{array}$ \\
\hline 3.6 & 19.9 & $1.4 \times 10^{42}$ & \\
4.5 & 12.8 & $7.2 \times 10^{41}$ & \\
5.8 & 24.4 & $1.1 \times 10^{42}$ & \\
8.0 & 51.8 & $1.6 \times 10^{42}$ & \\
24 & 49.4 & $5.2 \times 10^{41}$ & \\
70 & 666. & $2.4 \times 10^{42}$ & \\
160 & 1909. & $3.0 \times 10^{42}$ & \\
TIR & & $6.8 \times 10^{42}$ & \\
H $\alpha$ & & $3.1 \times 10^{40}$ & $4.2 \times 10^{40}$ \\
FUV & & $3.9 \times 10^{42}$ & $7.0 \times 10^{42}$ \\
NUV & & $3.2 \times 10^{42}$ & $6.3 \times 10^{42}$ \\
\hline
\end{tabular}

and $24 \mu \mathrm{m}$ in Paper I was $1.0 \times 10^{9} L_{\odot}$, but within a shorter radius $(5 \mathrm{kpc})$, hence the two values are consistent. Using the values in Table 1, we show in Fig. 1 the mid- and far-IR spectral energy distribution (SED) together with IRAS data from Rice et al. (1990) which, given the uncertainties, turn out to be in satisfactory agreement with our measurements. In the lower panel of Fig. 1, the SED is depicted as $v I_{v}$ which can be directly compared with the IR SED of 71 nearby galaxies given by Dale et al. (2005). The SED results from two major components: the stellar photospheric emission, which decreases from 3.6 to longer wavelengths, and the rising continuum due to dust emission. The peak at $8.0 \mu \mathrm{m}$ gives clear evidence of the presence of PAHs in M33.

The integrated FUV and NUV luminosities within $7 \mathrm{kpc}$ are $3.9 \times 10^{42} \mathrm{erg} \mathrm{s}^{-1}$ and $3.2 \times 10^{42} \mathrm{erg} \mathrm{s}^{-1}$ respectively (Gil de Paz et al. 2007). The $\mathrm{H} \alpha$ luminosity (as given by Hoopes \& Walterbos 2000) is $3.1 \times 10^{40} \mathrm{erg} \mathrm{s}^{-1}$. Again, Table 1 summarises the total fluxes, and luminosities measured within the SF disk of M 33 before applying any extinction correction, and with extinction corrections derived in Sect. 4.1.

\subsection{IR radial profiles}

Previous work has suggested that the SF disk in M33 shows three morphologically distinct areas: first, the innermost $1 \mathrm{kpc}$ where a weak bar is in place and metallicity is enhanced; second, the inner $3.5 \mathrm{kpc}$ disk where spiral arms and GMCs are prominent; and finally the outer disk where structures weaken before the edge of the SF disk occurs at about $7 \mathrm{kpc}$. It is therefore of interest to examine how the old and young stellar populations and the dust emission vary in these three regions and in general as a function of radius.

To extract the IR radial profiles from images, we used the IRAF task ellipse, fixing the center to the nominal position of M $33\left(01^{\mathrm{h}} 33^{\mathrm{m}} 50^{\mathrm{s}} .90,+30^{\circ} 39^{\prime} 35^{\prime \prime}\right.$. 8$)$, the axial ratio to 0.59 as for a fixed disk inclination of $54^{\circ}$ and the position angle to $22.5^{\circ}$.

We show in Fig. 2 the elliptically averaged surface brightness $\left(I_{\mathrm{A}}\right)$ in the IRAC and MIPS bands, as a function of galactocentric radius after correcting flux densities to face-on values. In Fig. 2, radial bins increase logarithmically: the multiplicative factor between two consecutive elliptical semiaxes is 1.1 , which is an increment of $10 \%$. The errors are computed by quadratically adding the error of the mean along the ellipse provided by the ellipse task itself and the error on the subtracted background. This background error is estimated for each image by considering the noise and flatness of the image in the most peripheral
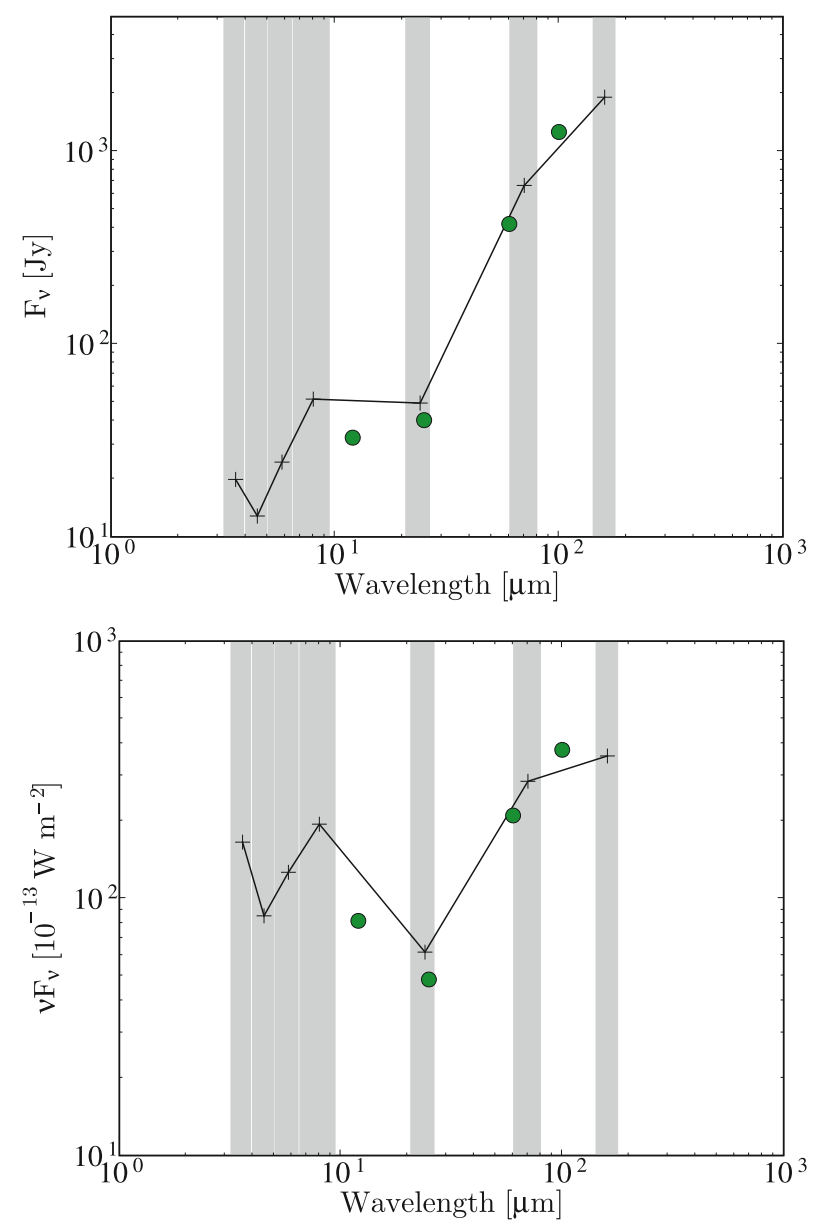

Fig. 1. The infrared spectral energy distribution of M 33 is depicted by (black) pluses (IRAC and MIPS Spitzer data). The IRAS data (12, 25, 60 , and $100 \mu \mathrm{m}$, green circles) from Rice et al. (1990) are compatible with our data within the quoted uncertainties.

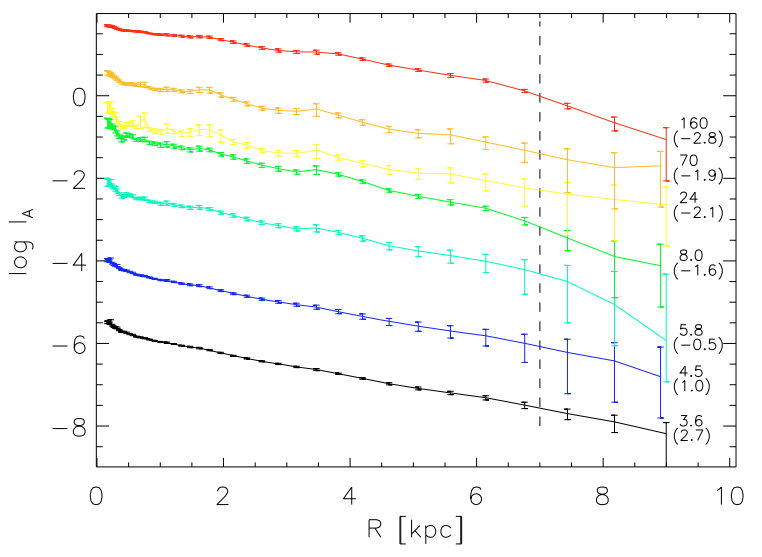

Fig. 2. Radial profiles, using logarithmic bins, of the Spitzer MIPS+IRAC surface brightness in arbitrary units. For clarity, results for the different bandpasses have been shifted in the vertical direction (within brackets, the logarithmic shift to be added to the ordinates to obtain the real surface brightness in $\mathrm{mJy} \mathrm{pc}^{-2}$ ). The dashed vertical line at $7 \mathrm{kpc}$ marks the edge of the star-forming disk, see text.

areas (considering 30 different areas around the borders). These estimates of the background uncertainty agree with the level of oscillation of the mean intensity from ellipse when it achieves background flux levels. 
Table 2. Scale lengths in kpc for the radially declining surface brightness at various IR wavelengths.

\begin{tabular}{cccc}
\hline \hline Wavelength & $0.5: 3.5 \mathrm{kpc}$ & $3.5: 7 \mathrm{kpc}$ & $0.5: 7 \mathrm{kpc}$ \\
\hline $3.6 \mu \mathrm{m}$ & $1.51 \pm 0.02$ & $1.61 \pm 0.05$ & $1.56 \pm 0.01$ \\
$4.5 \mu \mathrm{m}$ & $1.52 \pm 0.04$ & $1.60 \pm 0.15$ & $1.55 \pm 0.03$ \\
$5.8 \mu \mathrm{m}$ & $1.59 \pm 0.06$ & $1.36 \pm 0.13$ & $1.61 \pm 0.04$ \\
$8.0 \mu \mathrm{m}$ & $1.52 \pm 0.07$ & $1.21 \pm 0.05$ & $1.44 \pm 0.02$ \\
$24 \mu \mathrm{m}$ & $1.71 \pm 0.10$ & $1.59 \pm 0.20$ & $1.77 \pm 0.06$ \\
$70 \mu \mathrm{m}$ & $1.78 \pm 0.10$ & $1.55 \pm 0.14$ & $1.74 \pm 0.05$ \\
$160 \mu \mathrm{m}$ & $2.41 \pm 0.09$ & $1.48 \pm 0.04$ & $1.99 \pm 0.02$ \\
\hline
\end{tabular}

The extraction of the IRAC and MIPS-24 profiles was performed on images where foreground stars had previously been removed; we observed, that they introduce significant local enhancement in the profiles, particularly at 3.6 and $4.5 \mu \mathrm{m}$, the most sensitive to photospheric emission. At all wavelengths, the average surface brightness shows an enhancement in the innermost $0.5 \mathrm{kpc}$ region (see Sect. 3.3), then declines outwards exponentially. While the old stellar population dominates at short wavelengths, PAHs and Very Small Grains (VSGs) in the surroundings of hot stars are expected to contribute at 8 and $24 \mu \mathrm{m}$ respectively. Hence these last profiles display more features, especially in the inner disk where spiral arms are prominent. These are visible also in the emission at $70 \mu \mathrm{m}$, while at $160 \mu \mathrm{m}$ the coarse resolution limits the detection of such features in the elliptical averages.

The $\chi^{2}$ fit exponential scale lengths fitted from $0.5 \mathrm{kpc}$ to the edge of the star-forming disk are reported in Table 2 . The scale lengths are computed after rebbining the data into radial bins of equal size ( 50 pixels i.e. $0.24 \mathrm{kpc}$ ) taking into account the relative brightness uncertainties. All MIPS scale lengths are, within $3 \sigma$, compatible with those determined by Tabatabaei et al. (2007b, see their Table 7). The profiles at 3.6, 4.5, 24, and $70 \mu \mathrm{m}$ are consistent with a single exponential scale length throughout the star-forming disk. This is $\sim 1.55 \mathrm{kpc}$ for the old stellar population emission and $\sim 1.75 \mathrm{kpc}$ for the dust emission at 24 and $70 \mu \mathrm{m}$. Two different slopes for the inner $(0.5-3.5 \mathrm{kpc})$ and outer (3.5-7.0 kpc) star-forming disk are found instead at 5.8, 8.0 and $160 \mu \mathrm{m}$. At these wavelengths the IR brightness declines more steeply beyond $3.5 \mathrm{kpc}$ and drops at the edge of the star-forming disk. Decreases at the disk edge are less evident at other wavelengths, even though the uncertainties at large radii make the data consistent either with the no-drop hypothesis or with the drop that undoubtedly exists, namely at $160 \mu \mathrm{m}$. We also note that the disk of M 33 starts warping beyond $7 \mathrm{kpc}$, which adds uncertainties which have not been taken into account.

The similarity of the 5.8, 8.0, and $160 \mu \mathrm{m}$ emission may be understood by comparing radial trends of PAH emission and cold dust. PAHs contribute to the $5.8 \mu \mathrm{m}$ emission and even more to the $8.0 \mu \mathrm{m}$ emission band. Haas et al. (2002) and Bendo et al. (2008) have shown that PAH and cold dust emission correlate spatially and inferred that the diffuse ISRF is the power source of both emissions. Indeed, M 33 both at $8 \mu \mathrm{m}$ and $160 \mu \mathrm{m}$ shows a steeper decline in the outer disk. This might be due to a decrease in the surface density of the 8 and $160 \mu \mathrm{m}$ carriers since the decrease of the energy density of the ISRF in the outer disk is not steeper (see also Sect. 3.3). However, the $8 \mu \mathrm{m}$ profile declines with a steeper slope throughout the whole disk implying that the 8 to $160 \mu \mathrm{m}$ ratio also declines as we move outwards. One possibility is that the PAH abundance or emission is more sensitive to quantities which vary across the disk, such as the metallicity, the efficiency of supernovae shocks in

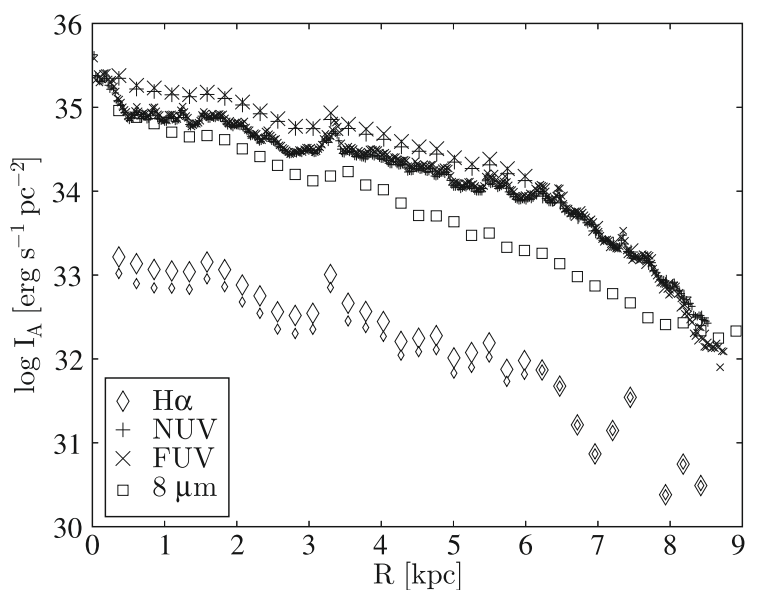

Fig. 3. Brightness radial profiles in the FUV (small crosses), NUV (small pluses) and $\mathrm{H} \alpha$ (small diamonds). Extinction corrected brightness profiles in bins of $0.24 \mathrm{kpc}$ are also shown in the FUV (large crosses), NUV (large pluses) and $\mathrm{H} \alpha$ (large diamonds). $I_{\mathrm{A}}$ is here converted to an absolute surface luminosity scale, that is multiplied by $v \times 4 \pi D^{2}$. The $8 \mu \mathrm{m}$ radial profile (squares) is plotted for comparison with the UV brightness.

destroying the molecules (O'Halloran et al. 2006), or the disk gravity, which affects the cooling and ionisation balance in the ISM (see Sect. 3.4). M 33 has a shallow metallicity gradient, and the oxygen abundance $[\mathrm{O} / \mathrm{H}]$ is always above -3.8 , except at the edge of the star-forming disk (Magrini et al. 2007b). This casts some doubt that the metallicity is the main driver of the fast radial decline of PAH emission.

The scale length inferred for the old stellar population using the 3.6 and $4.5 \mu \mathrm{m}$ emission is consistent with what earlier studies have derived in the inner part of the disk using photometry in the $K$-band (1.4 $\pm 0.1 \mathrm{kpc}$, Regan \& Vogel 1994). Ground based near IR data for this galaxy does not extend radially outwards as far as Spitzer data, but a colour map of the 3.6-2.2 $\mu \mathrm{m}$ emission using the 2MASS $(\mathrm{J}+\mathrm{H}+\mathrm{K})$ image (Jarrett et al. 2003) reveals a radial gradient between 0.5 and $5 \mathrm{kpc}$ due to the smaller scale length of the $\mathrm{J}+\mathrm{H}+\mathrm{K}$ emission (of the order of $1 \mathrm{kpc}$ ). This might imply that intermediate age, cool supergiants contribute a substantial fraction of the NIR emission. The colour map follows the spiral morphology, suggesting that cool supergiants are more likely candidates than giants.

\subsection{UV and $\mathrm{H} \alpha$ line radial profiles}

To evaluate the recent SFR across M 33 we will use the FUV and $\mathrm{H} \alpha$ radial profiles. It is more reliable to compute the SFR from integrated emission across the disk rather than from single SF sites. This is because SF sites lose part of their energetic photons in the surrounding ISM and the stars themselves diffuse out of their birthplace over time. FUV is a diagnostic of a somewhat younger (30-100 Myr) star formation than NUV (100-300 Myr), while H $\alpha$ relates to the youngest stellar population. In Fig. 3, we show the observed radial profiles of the NUV, FUV and $\mathrm{H} \alpha$ emissions (Thilker et al. 2005) in bins of $0.24 \mathrm{kpc} . \mathrm{H} \alpha$, FUV and NUV display very similar radial profiles which points to a scarce radial evolution of the SFR in the last 300 Myr. Unlike other galaxies (Thilker et al. 2007a), in M 33, the UV emission does not extend farther than $\mathrm{H} \alpha$. In addition, we observe no strong gradient in the colour ratio FUV-NUV; M 33 is only slightly bluer with increasing radius, which is opposite to the more pronounced trends found by Bianchi et al. (2005) in 
Table 3. Scale lengths for the $\mathrm{H} \alpha$, UV (uncorrected and corrected for extinction), the molecular and total gas emission.

\begin{tabular}{ccccc}
\hline \hline Line & Wavelength & $\begin{array}{c}\text { Range } \\
\mathrm{kpc}\end{array}$ & $\begin{array}{c}h_{\mathrm{r}} \\
\mathrm{kpc}\end{array}$ & $\begin{array}{c}h_{\mathrm{r}}(\text { corr }) \\
\mathrm{kpc}\end{array}$ \\
\hline $\mathrm{H} \alpha$ & $6563 \AA$ & {$[0.5: 6.0]$} & $2.0 \pm 0.2$ & $1.8 \pm 0.1$ \\
NUV & $1750-2750 \AA$ & {$[0.5: 6.0]$} & $2.3 \pm 0.1$ & $2.1 \pm 0.1$ \\
FUV & $1350-1750 \AA$ & {$[0.5: 6.0]$} & $2.4 \pm 0.1$ & $2.2 \pm 0.1$ \\
CO BIMA & & {$[0.5: 6.0]$} & $1.4 \pm 0.1$ & - \\
CO FCRAO & & {$[0.5: 6.0]$} & $2.4_{-0.4}^{+1}$ & - \\
Total gas & & {$[0.5: 6.0]$} & $16_{-5}^{+18}$ & - \\
\hline
\end{tabular}

M51 and M 101. This confirms the lack of an appreciable age gradient in the young stellar populations, and precludes strong radial variations of the UV spectrum.

UV and $\mathrm{H} \alpha$ show a strong enhancement in the innermost $0.5 \mathrm{kpc}$ likely due to the effect of the bar fueling the circumnuclear region (Corbelli \& Walterbos 2007). This is another reason why we excluded that region from our global fits. The radial scale lengths of the UV and $\mathrm{H} \alpha$ brightnesses are shown in the fourth column of Table 3 . They do not show significant variations between the inner and the outer disk and hence we display only their average values across the whole disk. In the last column of Table 3 we give these values after correcting for extinction, as described in the next section.

All three young SF tracers -H $\alpha$, FUV, and NUV- display a compatible scale length of about $2 \mathrm{kpc}$ while the oldest stellar populations (3.6 and $4.5 \mu \mathrm{m}$ ) exhibit a shorter one (approximately $1.5 \mathrm{kpc}$ ). The fact that the youngest stellar component has the longest scale length supports the inside-out disk growth scenario for M 33 (e.g. Magrini et al. 2007a). In general, our data suggest that dust emission at MIPS wavelengths in M 33 is also declining radially with longer scale lengths than the old stellar population, especially in the inner disk. The average dust emission scale length over the SF disk is consistent with the one traced by the young stellar populations even though the shorter scale length of the IR in the outer disk, especially at $160 \mu \mathrm{m}$, suggests a decline of the dust surface density.

\subsection{Atomic and molecular gas distributions}

In Fig. 4 we show the atomic and molecular gas column density as a function of galactocentric radius and Table 3 gives their radial scale lengths. The total gas distribution, sum of the H I (using the Arecibo data, except for the first four inner radii where we used the values from Westerbork) and of the diffuse molecular gas (using the FCRAO data), is also shown in Fig. 4. Uncertainties on the radial scale lengths for the CO-FCRAO and total gas are computed using the $\chi^{2}$ statistic taking into account the dispersion of the data in each radial bin. In Fig. 4 we display for clarity only the dispersions for the total gas surface density distribution, which is of relevance for this paper. The CO-BIMA scale length and its uncertainty are taken from Engargiola et al. (2003). The H I surface density is remarkably constant with radius (as already noted by Devereux et al. 1997), with a value of $\sim 10 M_{\odot} \mathrm{pc}^{-2}$, over most of the galaxy. Only beyond $6 \mathrm{kpc}$ is a cutoff observed in the Westerbork data, likely due to interferometer losses where the ISM is more uniformly diffuse. The situation is different for the molecular gas: the $\mathrm{CO}$ emission decreases steeply outwards, implying a less efficient conversion from atomic to molecular gas at larger radii. As discussed by Heyer et al. (2004), the molecular fraction is regulated by the

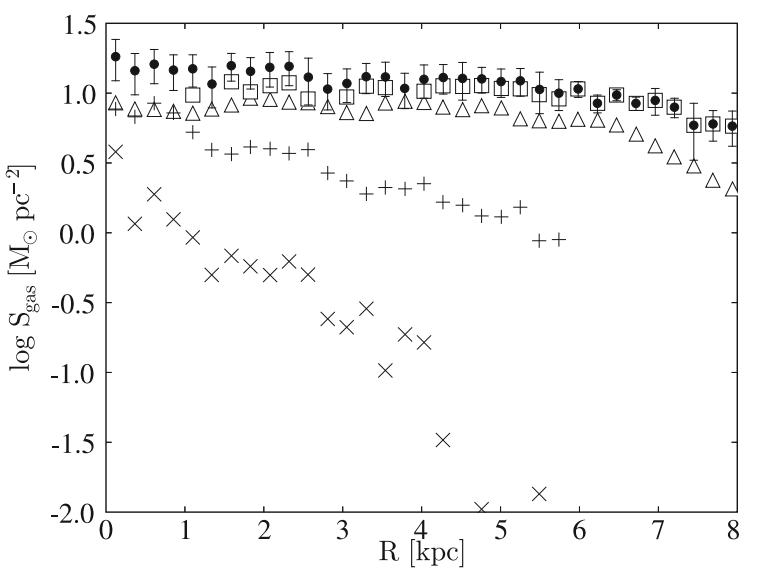

Fig. 4. Gas surface densities. The atomic gas data is shown by the open squares and open triangles for the Arecibo and Westerbork data, respectively. The molecular gas is shown by crosses and pluses for the BIMA and FCRAO data, respectively. The sum of the atomic (using the Arecibo data, except for the first four inner radii where we used the values from Westerbork) and molecular gas (FCRAO) is also displayed with filled circles. Error bars show the dispersion in each bin.

balance between formation rate, related to the hydrostatic pressure in the galactic plane, and dissociation rate related to the ISRF. The hydrostatic pressure decreases rapidly outwards because of the decrease of the stellar disk surface density, which dominates over the gas surface density inside $5 \mathrm{kpc}$ (Corbelli 2003).

\section{Dust in the disk}

\subsection{UV and Optical extinctions}

To correct the UV and $\mathrm{H} \alpha$ luminosities for internal extinction we adopt the prescription by Calzetti (2001) which relates $A_{\mathrm{V}}$ to the TIR and FUV luminosities:

$A_{\mathrm{V}}=C \times 1.76 \times \log \left(\frac{1}{1.68} \times \frac{L(\mathrm{TIR})}{L(\mathrm{FUV})}+1\right)$.

This gives an estimate of the average extinction of radiation from sources within the M 33 disk. The value of $C$ depends on whether we consider extinction of the stellar continuum or of the radiation from the ionised gas. In the model considered by Calzetti (2001), the dust is driven to the outer part of star-forming regions by first-generation massive stellar winds and supernova events. The dust will then produce a screen-like extinction for the radiation from central stars and from the ionised gas. Previous generations of stars, still contributing substantially to the UV emission, will instead be interspersed within the dust, since they tend to diffuse out from the central region. Consequently, the extinction of the stellar continuum from older stars will be lower than the extinction suffered by younger stars and by the ionised gas. In practice, $C=1$ for the ionised gas and $C=0.44$ for the stellar continuum. In the case of M 33 this scenario is supported by the distinct morphology of the GALEX and $\mathrm{H} \alpha$ maps: the H II regions are very localised, the UV much less so. However since about half of the $\mathrm{H} \alpha$ radiation in M33 is diffuse we shall consider an average value: $C=0.7$ for the ionised gas. This model assumes that the bulk of dust heating, which is re-radiated in the IR, comes from massive stars, the main contributors of UV photons. As we will see later in this paper, this assumption might not be valid for the MIR radiation at $24 \mu \mathrm{m}$ whose contribution to the TIR, however, is small, about $10 \%$. 


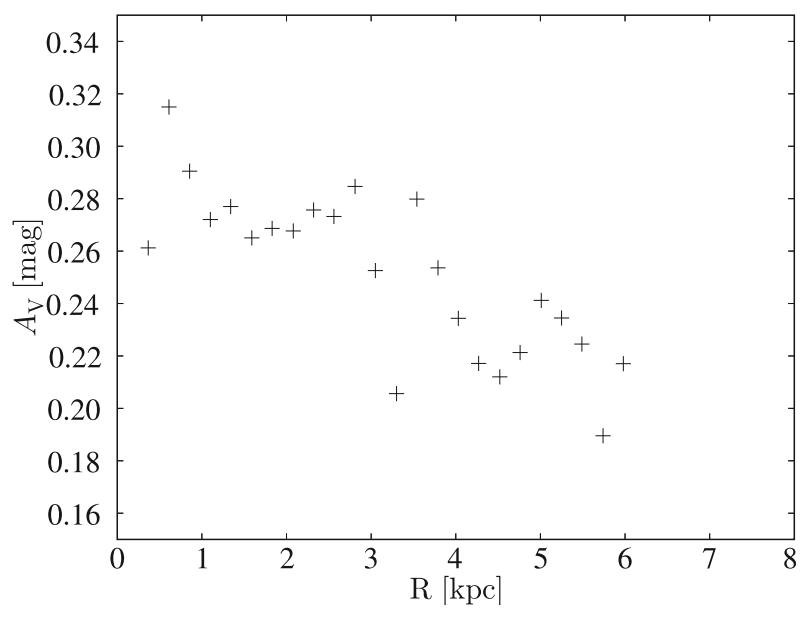

Fig. 5. $A_{\mathrm{V}}$ face-on values for the stellar continuum averaged in radial bins of $0.24 \mathrm{kpc}$, as a function of galactocentric radius.

Using a standard extinction curve, $A_{\mathrm{V}}=3.1 E(B-V)$, and following Seibert et al. (2005), i.e. $A_{\mathrm{FUV}}=8.29 E(B-V)$, we can infer $A_{\mathrm{FUV}}$. Globally, $A_{\mathrm{V}}$ is found to be $0.30 \mathrm{mag}$ (before applying face-on corrections) and hence $A_{\mathrm{FUV}}=0.53 \mathrm{mag}$, consistent with the findings of Buat \& Xu (1996) which give an upper limit of $0.7 \mathrm{mag}$; hence the extinction corrected FUV luminosity of M 33 is $6.3 \times 10^{42} \mathrm{erg} \mathrm{s}^{-1}$. Using $A_{\mathrm{NUV}}=8.18 \times E(B-V)$ (Seibert et al. 2005), $A_{\mathrm{NUV}}=0.52 \mathrm{mag}$ on average and the corrected NUV luminosity is $5.5 \times 10^{42} \mathrm{erg} \mathrm{s}^{-1}$.

In Fig. 5, we show the average face-on $A_{\mathrm{V}}$ value for the stellar continuum as a function of radius. $A_{\mathrm{V}}$ displays a rather constant value of about $0.27 \mathrm{mag}$ up to $3 \mathrm{kpc}$, then drops gently to below $0.22 \mathrm{mag}$ beyond $6 \mathrm{kpc}$. The drop of the UV luminosity at larger radii, due to the effective radial cut-off of the star forming disk, does not allow us to trace the extinction further out, where it may be considered negligible.

Extinction is found to be globally rather low, in agreement with Tabatabaei et al. (2007a). Nevertheless, extinction can be enhanced locally: in the cores of H II regions, values of $A_{\mathrm{V}} \sim 1 \mathrm{mag}$ are common (Massey et al. 1995; Devereux et al. 1997). The optical extinction, measured from the $\mathrm{H} \alpha$ to $\mathrm{H} \beta$ ratio in selected H II regions, is found to have a large scatter with an average of $0.65 \mathrm{mag}$ and with no clear radial dependence (Magrini et al. 2007b). Since, as noted above, the gas in H II regions should in principle suffer from a higher extinction than field stars, our average value of $0.25 \mathrm{mag}$ for the stellar continuum translates into $A_{\mathrm{V}} \simeq 0.57$ mag for the H II regions, very close to the finding of Magrini et al. (2007b).

From the radial profile of $A_{\mathrm{V}}$, we can recover the $\mathrm{UV}$ and $\mathrm{H} \alpha$ radial profiles corrected for extinction. These are shown in Fig. 3 and the relative scale lengths are given in Table 3 (FUV $=2.2 \mathrm{kpc} \pm 0.1 ; \mathrm{NUV}=2.1 \mathrm{kpc} \pm 0.1$; $\mathrm{H} \alpha=1.8 \mathrm{kpc} \pm 0.1)$. As it can be seen, extinction corrections are small and do not dramatically affect the profile slope. Upon correction for extinction at each radial bin, we find the total FUV luminosity to be $7.0 \times 10^{42} \mathrm{erg} \mathrm{s}^{-1}$. We deem this value more accurate than the $6.3 \times 10^{42} \mathrm{erg} \mathrm{s}^{-1}$ obtained above using the value of $A_{\mathrm{FUV}}$ averaged over the whole disk. Similarly, the total NUV luminosity radially corrected for extinction is $6.3 \times 10^{42} \mathrm{erg} \mathrm{s}^{-1}$. Following Calzetti (2001) we retain a global estimate of extinction for the ionized gas of $0.33 \mathrm{mag}$ at the $\mathrm{H} \alpha$ wavelength $(C=0.7)$. The corrected $\mathrm{H} \alpha$ luminosity is $4.2 \times 10^{40} \mathrm{erg} \mathrm{s}^{-1}$.

\subsection{Dust-to-gas ratio}

Since $A_{\mathrm{V}}$ is proportional to the dust column density, its ratio to the gas column density can be used to infer how the average dustto-gas ratio varies across the M 33 disk. As discussed above, extinction is higher towards $\mathrm{H}$ II regions than what Fig. 5 shows. Thus we expect the dust column density to be higher than what can be inferred from Fig. 5. There is also the possibility of an additional dust component dispersed in the ISM and not heated by the young stellar population, which has not been taken into account in the computation of $A_{\mathrm{V}}$.

In order to derive the dust column density from emission processes we shall derive the dust optical depth using the $160 \mu \mathrm{m}$ brightness and the dust temperature (see Sect. 4.3, for details on the dust temperature). The method used and the derived radial distribution of $\tau_{160}$, the optical depth at $160 \mu \mathrm{m}$, are very similar to those reported by Tabatabaei et al. (2007b) for the same galaxy. The opacity decreases by a factor 10 from the center to the edge of the star-forming disk. We then convert the dust opacity to the global visual extinction through half of the face-on disk of M 33 using:

$A_{\mathrm{V}}=\frac{1.086}{2} \times 2500 \times \tau_{160}$

Figure 6 shows the ratio of $A_{\mathrm{V}}$ to the total gas surface density as a function of radius using the $A_{\mathrm{V}}$ values derived both from Eqs. (2) and (3). The gas is the sum of the H I (from the Arecibo data, except the first point which is from Westerbork) and molecular gas (from the FCRAO data) ${ }^{3}$. There is a clear discrepancy: going radially outwards the dust-to-gas ratio derived from UV absorption does not show any clear dependence on radius. The dust-to-gas ratio derived from the IR emission decreases steadily. Clearly both methods have a caveat: the use of Eq. (2) is valid under the assumption that all dust is heated by UV radiation, in the converse case it overestimates $A_{\mathrm{V}}$. Also it depends on geometrical factors such as the clustering of hot stars, which emit the UV radiation. For the computation of $\tau_{160}$ we have assumed a single dust component in each radial bin (according to the temperatures shown in Fig. 9): in the presence of several temperature components the characteristic temperature one derives is biased to the higher values, resulting in an underestimate of the dust optical depth. Moreover the conversion factor between the optical depth in the IR and the one in the visual is highly uncertain: values range from 2000 to 5000 for our Galaxy (Whitcomb et al. 1981; Draine \& Lee 1984). The dust composition and size distribution in M33 can also be different from that in our Galaxy and can make precise calibration difficult (small particules and highly reflective materials will tend to increase the value of the conversion factor, e.g. Miyake \& Nakagawa 1993). Given these uncertainties, any conclusion about the average radial variations of the dust-to-gas ratio and of the dust optical depth in M 33 would be highly speculative.

\subsection{Dust temperature}

If the dust column density has a shallower radial decrease than the light profiles of the stellar population which is heating the dust, it is conceivable that the radial decrease of the 70 and $160 \mu \mathrm{m}$ emissions is due to a decrease of the dust heating radiation field rather than to a decrease of the dust column density. If

\footnotetext{
${ }^{3}$ If only interferometric data were used to estimate the $\mathrm{H}$ I density, due to the lower sensitivity and incomplete low spatial-frequency sampling, the dust-to-gas ratio would instead rise sharply in the outer part of the galaxy.
} 


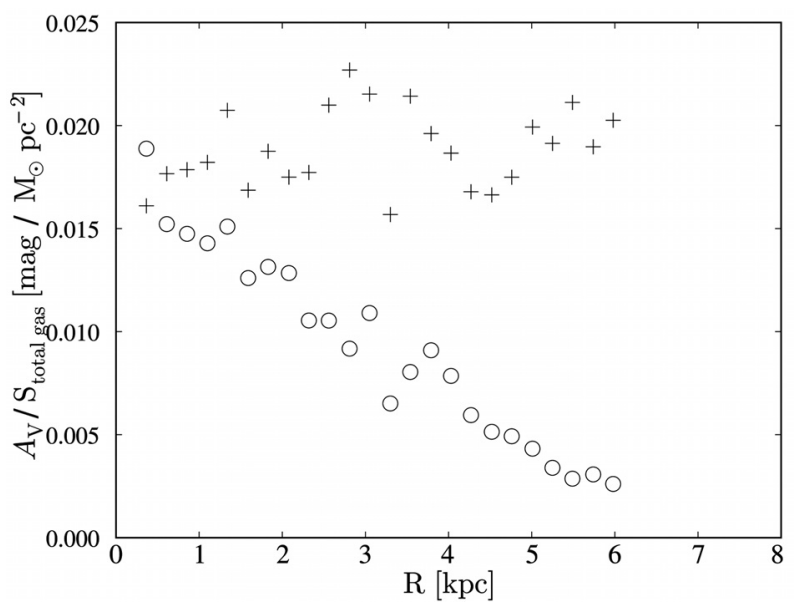

Fig. 6. The visual extinction normalized to the total gas column density as a function of the galactocentric radius. Plus symbols show this ratio for $A_{\mathrm{V}}$ derived from Eq. (2), open circles for $A_{\mathrm{V}}$ as from Eq. (3).

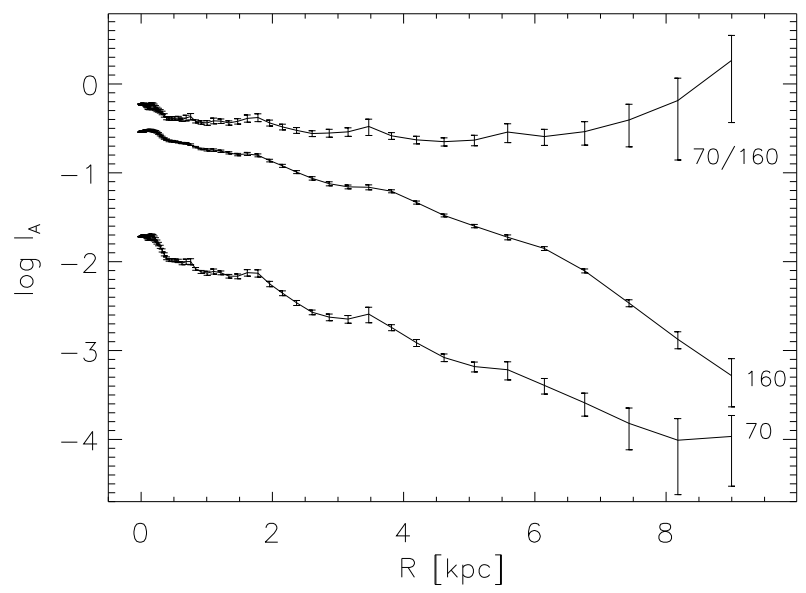

Fig. 7. Elliptically averaged 70 and $160 \mu \mathrm{m}$ surface brightness and their ratio as a function of the galactocentric radius, following logarithmic bins. The two brightness profiles are in arbitrary units and vertically displaced for clarity.

this picture is correct and the distribution of grain sizes does not change radially, any variation in the ratio of gas to 70 and $160 \mu \mathrm{m}$ emission is likely to be linked to dust temperature variations and cooler grains are expected in the outer part of the galaxy. The radial behaviour of the 70/160 colour is shown in Fig. 7.

There are clear oscillations, coinciding with the spiral pattern, where the colour becomes bluer due to the presence of large star-forming complexes. Overall this ratio decreases in the inner disk, where hot sources contribute substantially to the FIR emission, then remains constant up to the edge of the star-forming disk. The ratio flattens out in the outer disk: in fact the scale length of the $160 \mu \mathrm{m}$ emission, which was larger than that of the $70 \mu \mathrm{m}$ emission in the inner disk, decreases and emission at both wavelengths falls off radially in the same way. At $7 \mathrm{kpc}$ the data suggest an increase but the large error bars and the possible disk distortion due to the warp prohibit any definitive conclusion. By fitting an optically thin thermal spectrum $\left(\kappa_{\lambda} \propto \lambda^{-2}\right.$, see Hirashita et al. 2007, and references therein) to the elliptical averages at 70 and $160 \mu \mathrm{m}$, we derive the integrated FIR emission by integrating an optically thin thermal spectrum between 40 and $1100 \mu \mathrm{m}$, (which is the wavelength range which defines the FIR). The dust temperature, displayed in Fig. 9, decreases from $25 \mathrm{~K}$ in the innermost region to $21 \mathrm{~K}$ at about $4 \mathrm{kpc}$, then

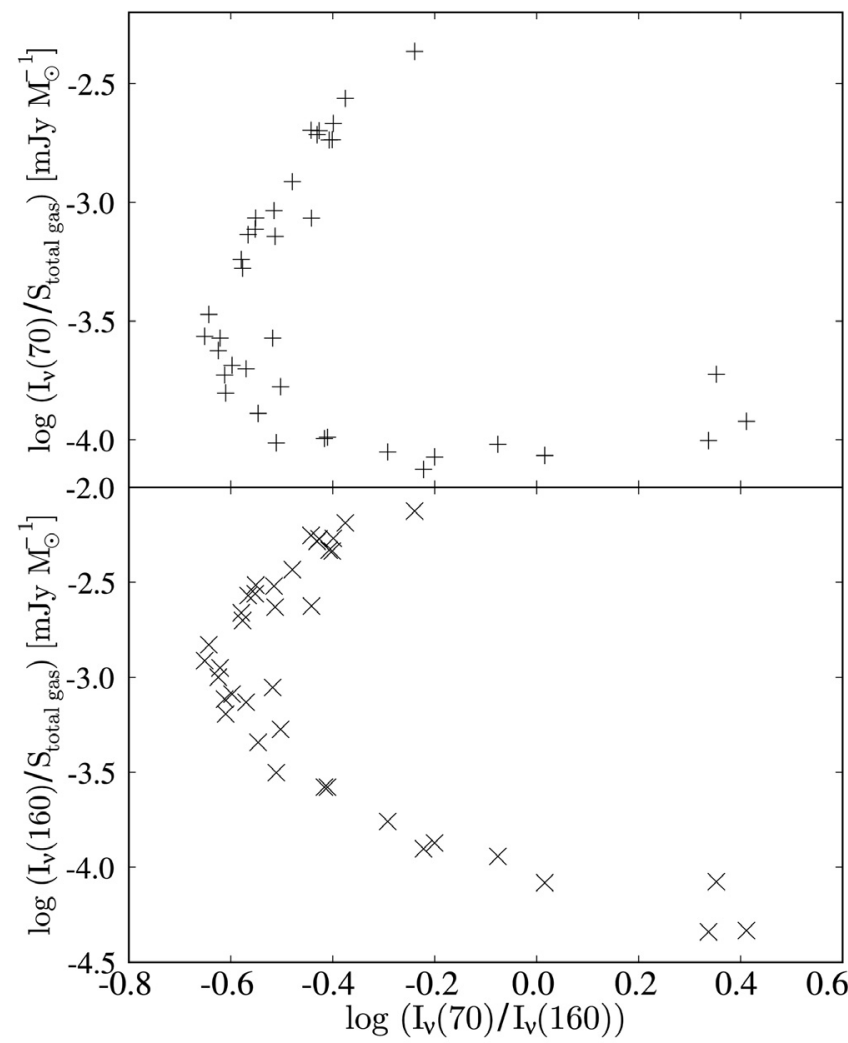

Fig. 8. Elliptically averaged FIR emission per unit gas versus FIR colour. They are displayed for the $70 \mu \mathrm{m}$ emission (upper panel) and for the $160 \mu \mathrm{m}$ emission (bottom panel). In both panels, the galactocentric radius increases from the upper to the lower points.

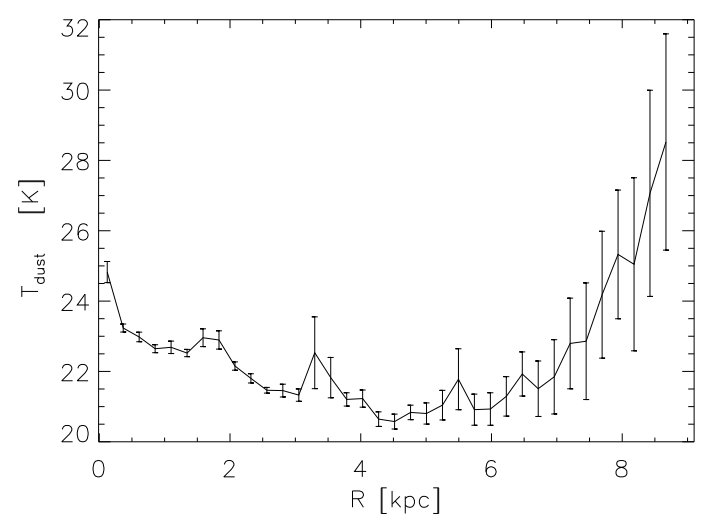

Fig. 9. Dust temperature as a function of radius, estimated by fitting a blackbody to the 70 and $160 \mu \mathrm{m}$ elliptical emissions, in bins of $0.24 \mathrm{kpc}$.

shows a flat plateau up to the edge of the star-forming disk where it increases. The integrated emission will be used in Sect. 6 to estimate the FIR luminosity and to derive the conversion factor between FIR luminosity and SFR.

Figure 8 shows the ratio of the 70 or $160 \mu \mathrm{m}$ surface brightness to the total gas column density as a function of the 70 to $160 \mu \mathrm{m}$ ratio. High values of the 70 or $160 \mu \mathrm{m}$ surface brightness to gas column density ratio correspond to the inner disk. Here the strength of the emission at 70 and $160 \mu \mathrm{m}$ is correlated primarily with dust heating and not with dust abundance since the FIR colour becomes redder as the dust emission per gas unit decreases. In the outer disk this is not so evident: the dust 
temperature stays constant out to the edge of the SF disk in the presence of a radially decreasing radiation field. If the dust column density does not change much and the UV radiation is the only heating source for grains, a change in the spectral energy distribution is required. But the lack of changes in the UV colour does not support this picture. A change in the dust abundance, as the steep radial decline of the $160 \mu \mathrm{m}$ emission seems to suggest, is more likely, assuming that the dust size distribution does not vary. There is however some anomaly in the distribution of evolved stars which suggests that the formation history of the M 33 disk has been rather complex and provides an alternative solution. An excess of carbon stars at large galactocentric radii has been found (e.g. Rowe et al. 2005; McQuinn et al. 2007; Cioni et al. 2008). Accretion events in the past brought some metal poor material beyond $4 \mathrm{kpc}$ thus favoring the production of carbon stars. The presence of additional radiation from those carbon-rich giants in the outer disk can heat the dust in their proximity thus providing an additional contribution to the $70 \mu \mathrm{m}$ surface brightness. We shall address better in the next section the role of evolved stars in powering the unresolved emission at shorter wavelengths. Some caution is needed here in drawing any definitive conclusion because at large radii, the $70 \mu \mathrm{m}$ image has a high noise level due to residual artifacts (see Paper I). Concerning possible changes in the grain size distribution we cannot fully constrain it with available data since the instruments are not sensitive to emission from a very cold dust component.

\section{Discrete sources and diffuse emission}

In this section, we derive the emission associated with discrete sources and compare this with the total emission in order to derive diffuse fractions as a function of radius. In general, M 33 is a galaxy with high diffuse fractions, the origin of which is still a subject of debate. The UV diffuse fraction of M 33 was studied by Thilker et al. (2005) to obtain indications on the location and nature of the non-ionising photons. The FUV diffuse fraction appears to be remarkably constant (equal to about 0.65 ) as a function of radius. Only beyond the edge of the star-forming disk is the slight increase of the FUV diffuse fraction that could indicate that some of the emission was not produced locally but rather scattered by dust. The $\mathrm{H} \alpha$ diffuse fraction was derived by Hoopes \& Walterbos (2000) and is about 0.4 throughout the SF disk.

In Paper I, we used the SExtractor software (Bertin \& Arnouts 1996) to compile a catalogue of 515 sources emitting at $24 \mu \mathrm{m}$. Following the process detailed in Paper I, we can extract sources at $8 \mu \mathrm{m}$, and in $\mathrm{H} \alpha$. The catalogue at $8 \mu \mathrm{m}$ comprises 516 sources, and the $\mathrm{H} \alpha$ catalogue 413 . The coarse resolution at 70 and $160 \mu \mathrm{m}$ allows the extraction of only the most prominent sources, about 150 in total; in any case, the emission associated with discrete sources would be highly underestimated at these longer wavelengths and we will not discuss them further.

Figure 10 illustrates the radial distributions of the integrated flux in discrete sources, in bins of $0.48 \mathrm{kpc}$. The upper panel shows that the IR flux at 8 and $24 \mu \mathrm{m}$ contributed by discrete sources declines radially more steeply than the total (scale lengths of $0.86 \pm 0.06$ and $0.88 \pm 0.13 \mathrm{kpc}$ for 8 and $24 \mu \mathrm{m}$ source number counts, respectively). This is not the case for the $\mathrm{H} \alpha$ sources, whose flux declines radially with a scale length of $1.5 \pm 0.3 \mathrm{kpc}$, practically the same as the total $\mathrm{H} \alpha$ emission. Similar results are found for the contribution of UV sources. Therefore, it is only the IR flux associated with discrete sources which shows a steep radial decline. The very high IR diffuse fractions in the outer part of the SF disk are responsible for

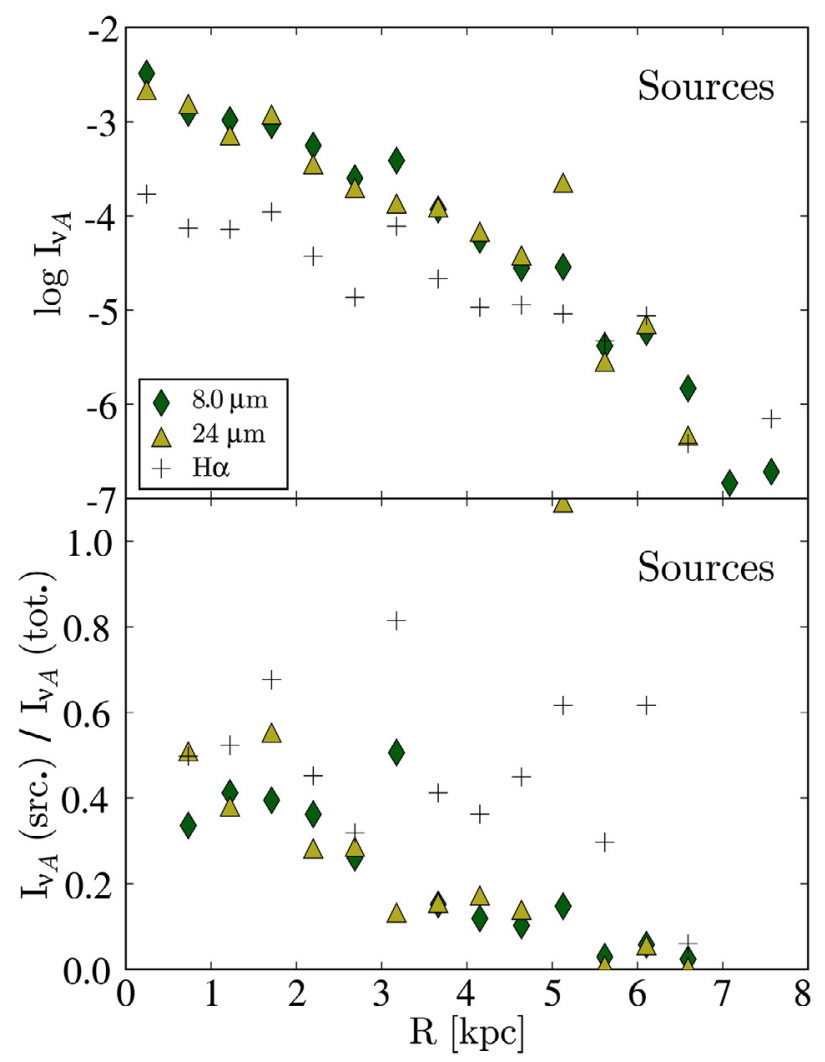

Fig. 10. Upper panel: infrared (in units of $\mathrm{mJy} \mathrm{pc}^{-2}$ ) and $\mathrm{H} \alpha$ (in units of $10^{13} \mathrm{erg} \mathrm{cm}^{-2} \mathrm{~s}^{-1} \mathrm{pc}^{-2}$ ) radial surface brightness distributions for discrete sources at $8 \mu \mathrm{m}$ (green diamonds), $24 \mu \mathrm{m}$ (yellow triangles) and $\mathrm{H} \alpha$ (black pluses). Bottom panel: fraction of the total fluxes due to emission from discrete sources at $8 \mu \mathrm{m}$ (green diamonds), at $24 \mu \mathrm{m}$ (yellow triangles) and in the $\mathrm{H} \alpha$ line (black pluses) as a function of radius.

the smooth decline of the light at IR wavelengths; no edge is seen, compared to the $\mathrm{H} \alpha$ and UV distributions which drop more sharply beyond $6 \mathrm{kpc}$.

The lower panel of Fig. 10 displays the results for the fraction of emission contributed by discrete sources with respect to the total. The $\mathrm{H} \alpha$ fraction shows some scatter but is nearly constant throughout the disk and equal to 0.5 on average; this is in agreement with Thilker et al. (2005) who, however, found a slight increase towards the center. The fraction of IR emission in discrete sources is nearly the same for the two wavelengths, but in the inner disc (up to $3.5 \mathrm{kpc}$ ) nearly $40 \%$ of the emission is contributed by discrete sources, while at larger radii they can account for less than $20 \%{ }^{4}$. The drop of the IR flux associated with discrete sources is beyond $3 \mathrm{kpc}$, where spiral arms appear to lose their strength. In the absence of strong perturbations, the large dusty SF complexes disappear and smaller sources reside in less opaque clumps (see also Corbelli et al. 2008 , in preparation).

\subsection{Sources and diffuse TIR}

We will now derive the radial trend for the TIR over the entire disk and for a sample of selected sources on the 8 and $24 \mu \mathrm{m}$ emissions. It is known that the relationship between $8,24 \mu \mathrm{m}$, and TIR luminosities depends on the properties of the galaxy

\footnotetext{
4 The discrepant point at $24 \mu \mathrm{m}$ just beyond $5 \mathrm{kpc}$ is due to the contribution of IC 133 .
} 


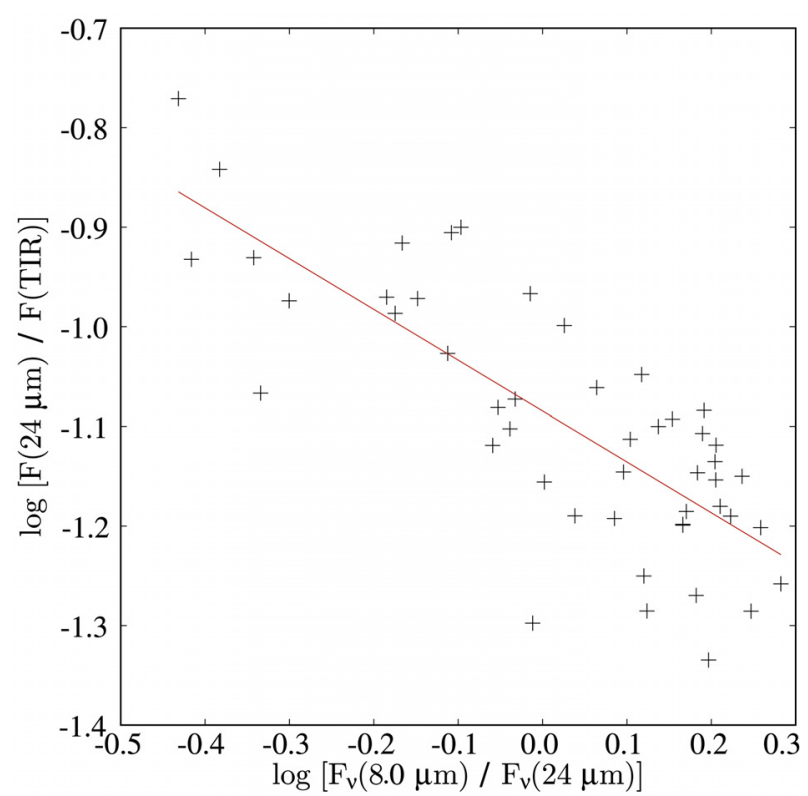

Fig. 11. The $24 \mu \mathrm{m}$ to TIR luminosity ratio as a function of the $8 \mu \mathrm{m} / 24 \mu \mathrm{m}$ flux ratio for 48 regions selected at $160 \mu \mathrm{m}$. The best linear fit to the data is shown by the plain (red) line.

considered and on the scale examined (e.g. Engelbracht et al. 2005; Pérez-González et al. 2006). We will first examine if the TIR emission in M33, associated with selected sources or with the overall disk emission, is correlated with the $8 \mu \mathrm{m}$ emission. For this purpose we extracted sources from the $160 \mu \mathrm{m}$ image and selected only those well fitted by a closely circular PSF (ellipticity $<0.3$ ). Then we measured them, within the aperture used at $160 \mu \mathrm{m}$, also at 8,24 , and $70 \mu \mathrm{m}$. We finally used the formula in Eq. (1) to estimate the TIR emission of 48 sources. The $24 \mu \mathrm{m}$ emission from discrete sources amounts to about $8 \%$ of their total TIR. Figure 11 shows the $24 \mu \mathrm{m}$ to TIR luminosity ratio as a function of the 8 to $24 \mu \mathrm{m}$ flux ratio. The best fit linear relation is:

$\log F($ TIR $)=\log F(24)+1.08+0.51 \log \left(\frac{F_{v}(8)}{F_{v}(24)}\right)$.

The $0.51 \pm 0.06$ coefficient is slightly lower than what Calzetti et al. (2005) derive in M 51 using a fixed and larger aperture size but similar to what Thilker et al. (2007b) derive for M 33 using a sample of IR selected clumps on scales of 160 pc. Figure 11 shows that a correlation exists but also that the third term in the above equation is small. This implies that the $8 \mu \mathrm{m}$ emission from sources depends on their TIR emission but does not contribute appreciably to it, as expected when dust is not very hot and PAH features dominate the $8 \mu \mathrm{m}$ band. This result only holds for bright IR sources, those well visible, resolved and isolated in the $160 \mu \mathrm{m}$ map; the same might not apply to fainter sources.

Using the radial profiles we can also derive the fit to the same relation but for the total emission (sources + diffuse component); we find :

$$
\log I_{\mathrm{A}}(\mathrm{TIR})=\log I_{\mathrm{A}}(24)+1.13+0.06 \log \left(\frac{I_{v \mathrm{~A}}(8)}{I_{v \mathrm{~A}}(24)}\right) .
$$

The correlation between the TIR and the $8 \mu \mathrm{m}$ emissions is in this case nearly absent, opposite to the trend found for selected IR sources. The absence of such a correlation in the diffuse emission, that is away from bright $\mathrm{H}$ II complexes, is the result of a

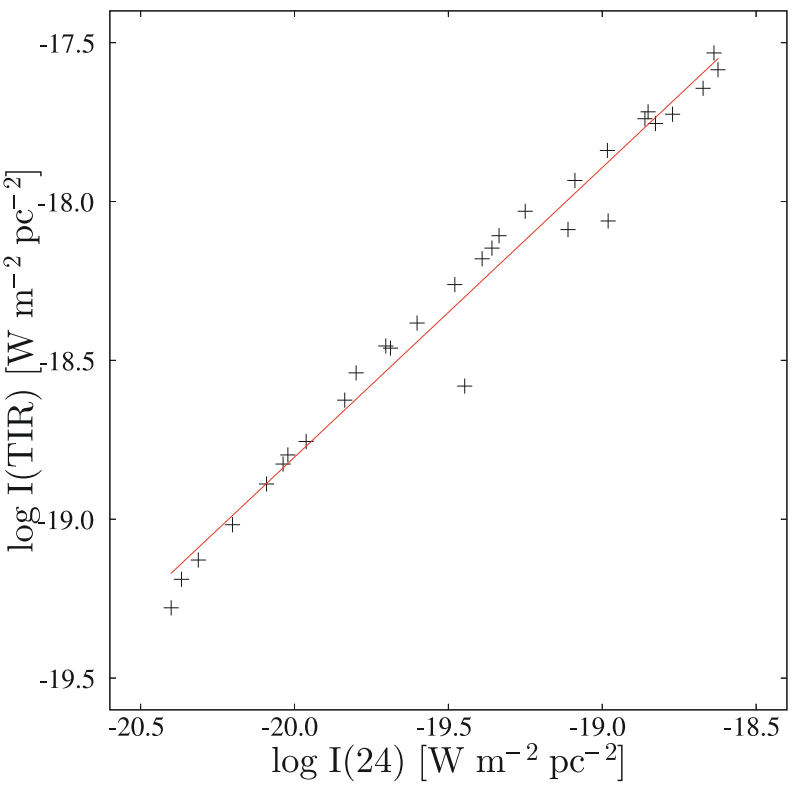

Fig. 12. TIR versus the $24 \mu \mathrm{m}$ emission, from radial averages. The slope of the best fitting linear relation between their logarithmic values (continuous line) is 0.91 .

different radial trend of the $8 \mu \mathrm{m}$ and of the IR emission at longer wavelength.

Figure 12 shows the remarkably good correlation, with a slope of about unity, between the TIR and the $24 \mu \mathrm{m}$ fluxes when using elliptical averages. This does not necessarily imply that the powering source in the two bands is the same. We shall see in the rest of this section that if two stellar populations of different ages are responsible for heating the dust in the TIR and $24 \mu \mathrm{m}$ band respectively, these results could imply that there has been no variation in the radial dependence of the SFR between the two epochs.

\subsection{Origin of the unresolved $24 \mu \mathrm{m}$ emission}

Discrete H II regions in the innermost half of the SF disk are clearly powering at least $40 \%$ of dust radiation at 24 and $8 \mu \mathrm{m}$. Then, what is left must be associated with dust spread out in the disk and heated by the diffuse ISRF or it must be associated with a "sea" of unresolved sources. The fraction of dust associated with $\mathrm{H}$ II regions decreases drastically with radius not only because the SFR per unit area is declining radially but also because they become dimmer in the IR beyond $3 \mathrm{kpc}$, as the drop in the bottom panel of Fig. 9 shows. This raises the problem of the energy requirement at these wavelengths. Grains associated with emission at 8 and $24 \mu \mathrm{m}$ wavelengths are thought to be VSGs, not necessarily spatially coincident with the large-size grains responsible for $160 \mu \mathrm{m}$ emission. Additionally, at $8 \mu \mathrm{m}$ the emission might have a large contribution from PAHs. We therefore test two different hypotheses for the powering of the diffuse emission at $24 \mu \mathrm{m}$ : (a) the interstellar radiation which is giving most of the power by stochastic heating of VSGs; $(b)$ evolved AGB stars, mostly carbon-type stars which are unresolved and have circumstellar dusty envelopes.

The first issue is the location of any unresolved $24 \mu \mathrm{m}$ emission. Radiation at this wavelength must be produced in situ and cannot be imputed to scattering of radiation emitted elsewhere. This is easily verified by considering the extremely low scattering cross section in the MIR. For a standard MRN dust model 
(Mathis et al. 1977) 5 and the optical constants by Laor \& Draine (1993) for the silicate material and by Zubko et al. (1996) for amorphous carbon, we can estimate the scattering coefficient at $24 \mu \mathrm{m}$ to be about $1.4 \times 10^{-6}$ times the extinction coefficient in the $V$ band. As we have seen, the face-on average $A_{V}$ value observed in the disk is about 0.25 , which translates into a total optical thickness of the disk, after correcting for scattering (Bianchi et al. 2005), $\tau_{V}=0.35$. This means that a $24 \mu \mathrm{m}$ photon may travel several Mpc in the M 33 disk before being scattered into our line of sight by dust. We note, incidentally, that the values observed for $A_{V}$ and for the total $\left(\mathrm{HI}+\mathrm{H}_{2}\right)$ gas column density $N_{\mathrm{H}}$ imply, for the dust model cited above, a mass density ratio $\rho_{\text {dust }} / \rho_{\mathrm{H}}=3.9 \times 10^{-3}$, slightly lower than in our Galaxy where estimates span from $4 \times 10^{-3}$ to $9 \times 10^{-3}$ (Whittet 1992), and in overall agreement with previous estimates, see Issa et al. (1990) and references therein.

\subsubsection{Emission of very small grains (VSGs)}

In the diffuse ISM, away from circumstellar environments, stochastic, or single-photon, emission by VSGs is thought to be the main MIR continuum-emission mechanism (e.g. Draine \& Li 2007). Precise computations are not easy to perform and in what follows we illustrate a model aimed at a rough estimate of the VSG contribution to the diffuse $24 \mu \mathrm{m}$ emission in M 33 .

The emissivity of a single grain when integrating over a time $\tau$ long enough to smooth out the impulsive behaviour will be

$\epsilon_{v}=\frac{\pi a^{2} Q_{v}^{\mathrm{abs}}}{\tau} \int_{0}^{\tau} B_{v}(T(t)) \mathrm{d} t$

where $Q_{v}^{\text {abs }}$ is the absorption efficiency of the grain and $B_{v}$ the Planck function at the grain temperature. We will model the temperature behaviour with time $T(t)$ of a certain grain as a stochastic sequence of identical, non-overlapping, flat-top events of height $T_{\mathrm{g}}$ and duration $\Delta t$, and $T=0$ otherwise. In each absorption event the grain will attain a temperature $T_{\mathrm{g}}$ which depends on the energy of the incoming photon and on the caloric properties of the grain. Given the photon, independently of the precise specific heat, the main factor will be the total number of atoms in the lattice since $T_{\mathrm{g}} \propto a^{-\delta}$, where $\delta$ is the dimensionality. Small grains are not expected to be spheres; they may be flat or plain needles or, more probably, a mixture of shapes and dimensions: we assume an average dimensionality $\delta=2$, i.e., planar. The grain will remain at temperature $T_{\mathrm{g}}$ for the time $\Delta t$ it takes to reradiate the absorbed energy. For a given incoming photon energy, $\pi a^{2}\left\langle Q_{\mathrm{IR}}^{\mathrm{abs}}\right\rangle T_{\mathrm{g}}^{4} \Delta t=$ const., where $\left\langle Q_{\mathrm{IR}}^{\mathrm{abs}}\right\rangle$ is an average IR efficiency. For the same illuminating field, the number of events per grain during $\tau \gg \Delta t$ is $N_{\mathrm{ev}} \propto \tau \pi a^{2}\left\langle Q_{\mathrm{UV}}^{\text {abs }}\right\rangle$, where $\left\langle Q_{\mathrm{UV}}^{\mathrm{abs}}\right\rangle$ is an average efficiency for the absorption of the heating photons. For small grains of radius $a<100 \AA$, we will always be in the Rayleigh regime and the absorption efficiency $Q_{v}^{\text {abs }} \propto a$. The characteristic grain temperatures we are interested in are those for which $\lambda F_{\lambda}$ peaks at $24 \mu \mathrm{m}$, that is $T_{\mathrm{g}} \simeq 150 \mathrm{~K}$. Around this temperature, the Planck function at $24 \mu \mathrm{m}$ can be approximated as a power law of $T$ by $B_{24}(T) \propto T^{3.9}$. Putting all this together in Eq. (6), it is easily derived that the $24 \mu \mathrm{m}$ emissivity of the single grain varies as $\epsilon_{24} \propto a^{3.2}$. This is in remarkably good agreement with simulations of the VSG emission in the range 10 to $50 \AA$ of radius (Kruegel 2003). Obviously this

\footnotetext{
$5 n(a) \propto a^{-3.5}, a_{\min }=0.001 \mu \mathrm{m}$ and $a_{\max }=0.3 \mu \mathrm{m}$, silicate and carbonaceous grains in equal number.
}

relation will hold only in the range of grain radii which can be heated up to $\sim 150 \mathrm{~K}$ by single-photon absorptions. The grains to be taken into consideration when heated by photons in the range $900<\lambda<5000 \AA$ are those containing roughly between 100 and 600 atoms, which translates into $15<a<40 \AA$.

Following this reasoning, upon averaging in time and composition, we estimate the emissivity of a $a=40 \AA$ grain in the local ISRF to be $\epsilon_{24}=4.3 \times 10^{-31} \mathrm{erg} \mathrm{s}^{-1} \mathrm{~Hz}^{-1} \mathrm{sr}^{-1}$. If we normalize the grain emission to this value and then integrate over the MRN size distribution between 10 and $50 \AA$ we can derive a volume emissivity and, given the observed gas-to-dust ratio, obtain the surface brightness at $24 \mu \mathrm{m}$ contributed by VSGs which, in this scheme, will be simply proportional to the gas column density and to the ISRF: $\sigma_{24}^{\mathrm{VSG}}=6.0 \times 10^{-6} \sigma_{\mathrm{H}} U \mathrm{mJy} \mathrm{pc}^{-2}$, where $\sigma_{\mathrm{H}}$ is the $\mathrm{H}$-gas column density in $M_{\odot} \mathrm{pc}^{-2}$, and $U$ the ratio between the actual ISRF and the one in the solar neighborhood 6 .

In order to estimate the factor $U$, we derive in Appendix $\mathrm{A}$ an approximate solution for a disk-like atmosphere which allows us to derive the field at the midplane, given the surface brightness seen by an external observer. We will use this method to estimate the M 33 ISRF at various wavelengths and compare it to the local values in the Galaxy (Weingartner \& Draine 2001).

Since the $3.6 \mu \mathrm{m}$ waveband is thought to directly measure the evolved stars' photospheric emission, we may use it as a proxy of the overall stellar content and therefore of the ISRF itself. Given the stellar population responsible for the $3.6 \mu \mathrm{m}$ emission, we postulate a ratio between dust and star scale heights $\beta=1 / 3$; in M33 at this wavelength we estimate the optical half-thickness $\tau_{0} / \mu \simeq 0.02$. With these assumptions, at $4 \mathrm{kpc}$ in the outer disk of M 33, the observed $3.6 \mu \mathrm{m}$ surface brightness implies a midplane ISRF which is 0.31 times the local Galactic value. Alternatively, since the VSG stochastic emission is powered mainly by UV photons, we may directly estimate the ISRF from the GALEX data. We assume $\beta=1 / 1$ and $\tau_{0} / \mu \simeq 0.9$ for both the NUV and FUV emission and in both cases we consistently derive, at $4 \mathrm{kpc}$, an ISRF 0.50 times the one in the solar neighborhood, in overall agreement with the $3.6 \mu \mathrm{m}$ result. If we then assume that the ISRF in the outer M 33 disk is about half the local Galactic value, we find that the predicted VSGs emission falls short by a factor $>10$ of the diffuse $24 \mu \mathrm{m}$ flux actually measured $^{7}$. Although exemplified here at a fiducial radius of $4 \mathrm{kpc}$, this is true at all radii, as well as for the total, unresolved $24 \mu \mathrm{m}$ luminosity. We have verified that this result depends weakly on the limits used for the range of grain radii and on the slope of the grain size distribution. Also, the VSG emission of spherical grains will be lower than what we have estimated here for flat $(\delta=2)$ geometries.

\subsubsection{Carbon stars}

To better understand the origin of the diffuse $24 \mu \mathrm{m}$ emission, we focus on a class of intermediate-age stars, the carbon stars. Carbon-rich giants, or carbon stars (CSs) are stars of low to intermediate initial mass, 0.8 to $4 M_{\odot}$ with extended dusty circumstellar envelopes mainly found in their asymptotic giant branch (AGB) evolutionary phase (Herwig 2005). 460 carbon stars have been observed using the Spitzer infrared spectrograph (IRS) in

\footnotetext{
${ }^{6}$ In the same units Draine \& $\mathrm{Li}$ (2007) obtain $\sigma_{24}^{\mathrm{VSG}} \simeq 2.0 \times$ $10^{-5} \sigma_{\mathrm{H}} U \mathrm{mJy} \mathrm{pc}^{-2}$.

7 Or a factor $>3$ when adopting the more generous emissivities by Draine \& Li (2007).
} 
the Magellanic Clouds by Groenewegen et al. (2007) and half of them in the LMC, an environment not dissimilar to the M 33 disk. While their $\lambda F_{\lambda}$ peaks around $8 \mu \mathrm{m}$, they still show rather strong emission at longer wavelengths, with a $\lambda F_{\lambda}$ at $100 \mu \mathrm{m}$ comparable to the one in the $\mathrm{J}$ bandpass. The observed spread in luminosity covers about 1.7 dex with the most luminous ones being the reddest, as expected in the case of AGB stars; when set at the distance of M 33, the average $24 \mu \mathrm{m}$ flux of a single CS is $F_{24}=0.21 \mathrm{mJy}$.

In order to estimate the possible contribution to the MIR emission by CSs we will make use of the Padova evolutionary tracks by Girardi et al. (2000) for a metallic content $Z=0.004^{8}$. It is during the shell helium-burning phase that stars climb the red supergiant branch in the HR diagram by increasing their luminosity and lowering the effective temperature. The duration of this AGB phase varies with the initial mass, from about $10^{7}$ to $10^{6} \mathrm{yr}$ in the CS mass range. Similarly, the luminosity attained on the AGB depends on mass but three quantities are roughly constant with mass: (1) the effective temperature range from $T_{\text {ini }} \simeq 4500$ to $T_{\text {fin }} \simeq 3800 \mathrm{~K}$; (2) the ratio between final and initial luminosity $L_{\mathrm{fin}} / L_{\mathrm{ini}} \simeq 13$ (that is, in $\log -\log$ plots, different masses evolve along identical, vertically displaced, parallel tracks); and (3) the bolometric energy release $\int_{\mathrm{AGB}} L \mathrm{~d} t \simeq 4.5 \times 10^{9} L_{\odot} \mathrm{yr}$.

If $L\left(m, t_{\mathrm{A}}\right)$ is the bolometric luminosity of a CS of initial mass $m$ after a time $t_{\mathrm{A}}$ since the entrance in AGB phase, we can define a time-averaged luminosity at mass $m$ :

$\langle L(m)\rangle=\frac{\int_{0}^{\tau_{\mathrm{A}}(m)} L\left(m, t_{\mathrm{A}}\right) \mathrm{d} t_{\mathrm{A}}}{\tau_{\mathrm{A}}(m)}$

where $\tau_{\mathrm{a}}(m)$ is the AGB phase duration for mass $m$. Given an initial mass function (IMF), we can derive an overall mean luminosity for all CSs:

$\langle\langle L\rangle\rangle=\frac{\int_{0.8 M_{\odot}}^{4.0 M_{\odot}}\langle L(m)\rangle m^{-\alpha} \mathrm{d} m}{\int_{0.8 M_{\odot}}^{4.0 M_{\odot}} m^{-\alpha} \mathrm{d} m}$

where $\alpha$ is the exponent of the IMF power law. Hereafter we adopt $\alpha=2.3$ for $0.5 \leq m \leq 100 M_{\odot}$ (Salpeter 1955) and 1.3 for $0.1 \leq m \leq 0.5 M_{\odot}$ (Kroupa 2001). For this IMF and the Padova tracks, we obtain $\langle\langle L\rangle\rangle=580 L_{\odot}$.

As we said, based on the observations in the LMC, we know the average expected $24 \mu \mathrm{m}$ flux from a CS in M33, and we may think of this as a mass and time average over the whole CS population, as outlined above for the luminosity, that is $\left\langle\left\langle F_{24}\right\rangle\right\rangle=0.21 \mathrm{mJy}$. We also noted that the effective temperature range of $\mathrm{CS}$ is rather restricted and quite similar for all the relevant masses. Given this circumstance, we may assume that with a good approximation $\left\langle\left\langle F_{24}\right\rangle\right\rangle \propto\langle\langle L\rangle\rangle$ with a proportionality constant $\left\langle\left\langle K_{24-L}\right\rangle\right\rangle=\left\langle\left\langle F_{24}\right\rangle\right\rangle /\langle\langle L\rangle\rangle=3.65 \times 10^{-4} \mathrm{mJy} L_{\odot}{ }^{-1}$.

At this point, if $F_{24}\left(m, t_{\mathrm{A}}\right)$ is the flux from the single CS, from a given region we expect a flux

$\Sigma_{24}=\int_{0.8 M_{\odot}}^{4.0 M_{\odot}} \dot{N}(m)\left\{\int_{0}^{\tau_{\mathrm{A}}(m)} F_{24}\left(m, t_{\mathrm{A}}\right) \mathrm{d} t_{\mathrm{A}}\right\} \mathrm{d} m$

where $\dot{N}(m)$ is the differential star-formation rate in that region, admitting it is constant with time (both integrally and

8 The tracks do not cover the full AGB phase but only up to the onset of thermal pulses. In this respect, our estimates constitute a lower limit to the CS contribution; due to the short duration of the pulsing phase, the correction is of little importance. in mass distribution). If we now assume that $F_{24}\left(m, t_{\mathrm{A}}\right)=$ $\left\langle\left\langle K_{24-L}\right\rangle\right\rangle L\left(m, t_{\mathrm{A}}\right)$, the theoretical tracks can be used to evaluate Eq. (9) and we obtain $\Sigma_{24}=2.05 \times 10^{5} \dot{M}_{T}$ mJy, where $\dot{M}_{T}$ is the total (from 0.08 to $100 M_{\odot}$ ) SFR in the region of interest in $M_{\odot} \mathrm{yr}^{-1}$. Note that, despite the rather strong assumption of proportionality between $F_{24}\left(m, t_{\mathrm{A}}\right)$ and $L\left(m, t_{\mathrm{A}}\right)$, the final result relies on estimates of $\int L \mathrm{~d} t$ along the AGB phase which, as said above, are quite robust and constant.

It turns out that the SFR required to explain the $24 \mu \mathrm{m}$ unresolved emission in M33 with the sole contribution of CSs is compatible with what is computed by the usual SFR indicators, as will be seen in the following. Note also that the most luminous CSs could well have been counted among the faint discrete sources, and that a small fraction of carbon stars might not have dusty shells (McQuinn et al. 2007). As a result the $24 \mu \mathrm{m}$ unresolved emission due to CS should give a lower SFR than the effective one. The total unresolved $24 \mu \mathrm{m}$ flux of M 33, 35 Jy, translates into a global SFR of $0.17 M_{\odot} \mathrm{yr}^{-1}$; the surface brightness in the outer disk at $4 \mathrm{kpc}$ is $\sim 4 \times 10^{-4} \mathrm{mJy} \mathrm{pc}^{-2}$ requiring $\dot{M}_{T} \simeq 2.0 M_{\odot} \mathrm{Gyr}^{-1} \mathrm{pc}^{-2}$.

\section{Star formation rates}

How well tracers at different wavelengths measure the actual SFR has been examined by a number of papers based on Spitzer data (e.g. Calzetti et al. 2005; Kennicutt et al. 2007, for M 51). In this section, we compare the SFRs derived from emission at different wavelengths as a function of the galactocentric radius (in bins of $0.24 \mathrm{kpc}$ ) and integrated over the whole SF disk. To compute the SFR from $\mathrm{H} \alpha$ and UV luminosities corrected for extinction (see Sect. 3) we use the stellar population synthesis model in Starburst 99 (Leitherer et al. 1999), for a continuous star-formation model with solar metallicity and the IMF cited in the previous section.

\subsection{SFR from $H \alpha$ and UV wavelengths}

The conversion between $\mathrm{H} \alpha$ luminosities and SFR given by the stellar population model is

$\mathrm{SFR}(\mathrm{H} \alpha)\left[M_{\odot} \mathrm{yr}^{-1}\right]=8.3 \times 10^{-42} L(\mathrm{H} \alpha)\left[\mathrm{erg} \mathrm{s}^{-1}\right]$.

This is within $5 \%$ of the value given by Kennicutt (1998), showing that the SFR dependence on $\mathrm{H} \alpha$ is rather insensitive to the assumptions about the lower-mass form of the IMF. The radial profile is shown in Fig. 13 and the total integrated SFR over the whole SF disk $(R<7 \mathrm{kpc})$ is $\operatorname{SFR}(\mathrm{H} \alpha)=0.35 M_{\odot} \mathrm{yr}^{-1}$ which correspond to the average SFR over about the last $10 \mathrm{Myr}$. To trace the SFR over a wider timescale, namely in the last $100 \mathrm{Myr}$, we convert the FUV luminosity according to the same population synthesis model at $1550 \AA$ as:

$\mathrm{SFR}(\mathrm{FUV})\left[M_{\odot} \mathrm{yr}^{-1}\right]=8.8 \times 10^{-44} L(\mathrm{FUV})\left[\mathrm{erg} \mathrm{s}^{-1}\right]$.

The above conversion (equivalent to $1.7 \times$ $10^{-28} L_{\nu}(\mathrm{FUV})\left[\mathrm{erg} \mathrm{s}^{-1} \mathrm{~Hz}^{-1}\right.$ ]) is also similar to the one given by Kennicutt (1998) (although we are using a slightly different IMF and wavelength range). The radial profile of the SFR derived from the FUV emission is shown in Fig. 13 and the SFR integrated over the SF disk is $\mathrm{SFR}(\mathrm{FUV})=0.55 M_{\odot} \mathrm{yr}^{-1}$, higher than the one derived from $\mathrm{H} \alpha$. The SFR declines radially with a scale length of $2.2 \pm 0.1 \mathrm{kpc}$ in the FUV and $1.8 \pm 0.1 \mathrm{kpc}$ in $\mathrm{H} \alpha$, a marginal difference considering the uncertainties. However there are two reasons why the two might indeed be different: one is because we are sampling two different 
SF epochs; the second is that $\mathrm{H} \alpha$ depends on the interstellar medium volume density and a radial decrease of this might suppress $\mathrm{H} \alpha$ and also induce a sharper $\mathrm{H} \alpha \mathrm{SF}$ edge (Elmegreen $\&$ Hunter 2006).

\subsection{SFR from IR wavelengths}

While in dusty environments IR-based SF tracers are quite reliable, in M33 this assumption is not obvious and requires caution in interpretation. Although within the single SF region most of the dust emission is powered by young stars, the situation is more complex when we integrate over the whole disk which, as said before, has an important diffuse fraction. In M 33 the emission at 70 and $160 \mu \mathrm{m}$ can be powered by the UV in the diffuse ISRF or by single H II regions: both cases are related to the presence of stars of young to moderate age. We expect, therefore, the FIR luminosity to be a valid SF tracer and yield a SFR similar to the one based on FUV data, once corrected for extinction. If we compute the FIR luminosity by fitting an optically thin $\left(\kappa_{\lambda} \propto \lambda^{-2}\right)$ thermal spectrum to the 70 and $160 \mu \mathrm{m}$ elliptical averages and then integrate this between 40 and $1100 \mu \mathrm{m}$ (see Sect. 4), we find that it correlates well with the TIR luminosity computed via Eq. (1). The average ratio between the TIR and the FIR luminosities is $1.40 \pm 0.04$ across the whole disk. The FIR emission thus modeled, integrated over the disk $(<7 \mathrm{kpc})$, amounts to $4.6 \times 10^{42} \mathrm{erg} \mathrm{s}^{-1}$.

If we choose the extinction corrected FUV as a reference for the SFR, we can derive the conversion factors for the other wavebands. We did this by using a $\chi^{2}$ minimization over the set of radial bins within $6 \mathrm{kpc}$, to avoid the most noisy data. For the FIR we obtain:

$\mathrm{SFR}(\mathrm{FIR})\left[M_{\odot} \mathrm{yr}^{-1}\right]=13 \times 10^{-44} L(\mathrm{FIR})\left[\mathrm{erg} \mathrm{s}^{-1}\right]$,

which is in the upper range of the values found by Buat \& Xu (1996) for late-type galaxies based on IRAS and UV flux measurements. A relatively high conversion factor is indicative of a relatively low dust content.

Using the same technique but for the TIR fluxes, we find that in order to match the FUV SFR:

$\mathrm{SFR}(\mathrm{TIR})\left[M_{\odot} \mathrm{yr}^{-1}\right]=8.8 \times 10^{-44} L(\mathrm{TIR})\left[\mathrm{erg} \mathrm{s}^{-1}\right]$.

As seen in Fig. 13, the FIR- and TIR-based SFR radial profiles show a smoother drop at the SF disk edge possibly due to their origin in more diffuse components, dust, and general ISRF.

If one is convinced that the $24 \mu \mathrm{m}$ also is powered by young stars, then normalizing the SFR based on the $24 \mu \mathrm{m}$ emission to the FUV SFR, one obtains:

$\operatorname{SFR}(24)\left[M_{\odot} \mathrm{yr}^{-1}\right]=1.2 \times 10^{-42} L(24)\left[\mathrm{erg} \mathrm{s}^{-1}\right]$.

This factor is about three times higher with respect to the relation given by Wu et al. (2005; see also Yun et al. 2001), which was derived by comparing IR and radio emission in the Spitzer First Look Survey Galaxies. They find:

$\operatorname{SFR}(24)\left[M_{\odot} \mathrm{yr}^{-1}\right]=3.9 \times 10^{-43} L(24)\left[\mathrm{erg} \mathrm{s}^{-1}\right]$.

Using this latter relation, the global SFR from $24 \mu \mathrm{m}$ emission in M 33 is only $0.2 M_{\odot} \mathrm{yr}^{-1}$ and the relative SFR per unit area is shown in Fig. 13. Equation (15) clearly underestimates the SFR. In the inner regions the $24 \mu \mathrm{m}$ and $\mathrm{H} \alpha$ SFRs follow the pattern of the galaxy: they are enhanced along the spiral arms and decrease monotonically away from the center. Beyond $4 \mathrm{kpc}$ the $24 \mu \mathrm{m}$ emission fraction linked to discrete sources drops. Diffuse

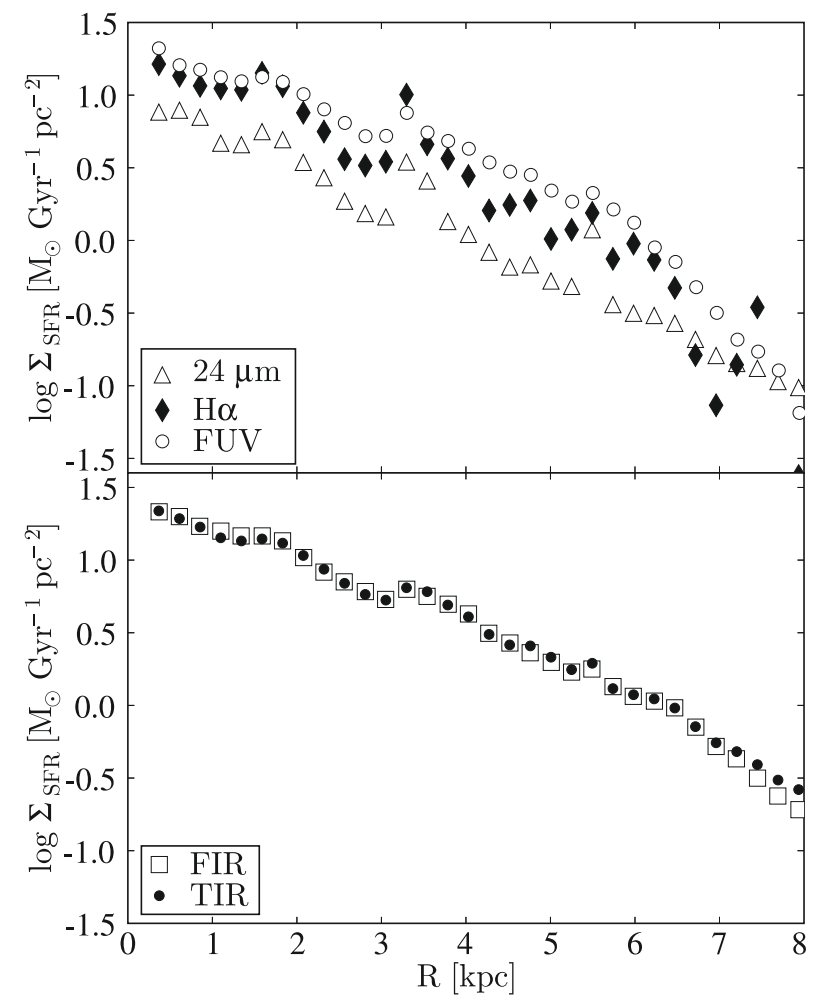

Fig. 13. SFR per square parsec as a function of radius using Eq. (11) for converting into SFR the FUV luminosity (open circles), Eq. (15) for the $24 \mu \mathrm{m}$ luminosity (open triangles), Eq. (10) for the $\mathrm{H} \alpha$ luminosity (filled diamonds) (upper panel), Eq. (11) for the FIR luminosity (open squares), and Eq. (13) for the TIR luminosity (filled circles) (bottom panel).

emission dominates which, as shown in the previous section, can be powered by more evolved stars and linked to the SFR about 1 Gyr ago rather than to recent episodes of SF. This explains the absence of any drop at the SF disk edge due to stellar diffusion of the more evolved population. Hence, the $24 \mu \mathrm{m}$ emission in late-type galaxies like M 33, with a few very large SF complexes and a large diffuse component, depends upon the presence of dusty envelopes around evolved stars. If the contribution in hot discrete sources is removed, it can give a lower limit to the SFR about 1 Gyr ago: the exact rate depends upon the fraction of evolved stars that have developed a dusty envelope.

\section{Summary and conclusions}

This second paper of the series focuses on various SFR diagnostics across the disk of the local spiral galaxy M33, and on the origin of the high IR diffuse emission. We investigate the radial trends of the IR emission at various wavelengths using Spitzer data, complementing these with GALEX data for the UV, with $\mathrm{H} \alpha$ data, and with data on the atomic and molecular gas distributions. Hereafter, we summarise our main results.

- The Spitzer radial profiles at 3.6, 4.5, 24, and $70 \mu \mathrm{m}$ are consistent with a single exponential scale length throughout the star-forming disk. This is $\sim 1.55 \mathrm{kpc}$ for the (old) stellar population emission and $\sim 1.75 \mathrm{kpc}$ for the dust emission at 24 and $70 \mu \mathrm{m}$. Two different slopes for the inner and outer starforming disk are found instead at 5.8, 8.0 and $160 \mu \mathrm{m}$. At these wavelengths the IR brightness declines more steeply beyond $3.5 \mathrm{kpc}$ and drops at the edge of the star-forming 
disk. The scale lengths more directly linked to SF diagnostics (H $\alpha$, FUV and NUV) are about $2 \mathrm{kpc}$, comparable to the IR ones in the inner disk, but longer than these in the outer star-forming disk. This implies that the dust column density is decreasing beyond $3.5 \mathrm{kpc}$, a trend confirmed by our estimate of the extinction from the TIR to FUV ratio. The $8 \mu \mathrm{m}$ emission is dominated by PAH features which show instead a faster decline with radius.

- Within the star-forming disk, that is within $7 \mathrm{kpc}$ of radius, we estimate the TIR, FUV, NUV, and $\mathrm{H} \alpha$ luminosities to be $6.8 \times 10^{42}, 7.0 \times 10^{42}, 6.3 \times 10^{42}$, and $4.2 \times 10^{40} \mathrm{erg} \mathrm{s}^{-1} \mathrm{re}-$ spectively after correcting for extinction. Extinction corrections are small and reflect the rather low dust content of the galaxy $\left(A_{\mathrm{V}} \sim 0.25\right.$, for stellar continuum averaged in rings of width $0.24 \mathrm{kpc}$ ). Extinction can be enhanced locally (cores of $\mathrm{H}$ II regions, for instance); however, the absence of large dusty complexes beyond $3 \mathrm{kpc}$, likely due to the weakness of the spiral pattern, implies that at large radii SF proceeds in smaller clumps with low extinction. Dust opacity derived from the $160 \mu \mathrm{m}$ emission under the assumption of single temperature component has a steeper radial decline than dust opacity inferred via the extinction model.

- A substantial amount of the emission of the M33 galaxy is in diffuse form at several wavelengths. The FUV diffuse fraction is equal to about 0.65 and does not depend on radius. The $\mathrm{H} \alpha$ diffuse fraction is more scattered but nearly constant throughout the disk of the galaxy and equals 0.5 on average. In the IR, at 8 and $24 \mu \mathrm{m}$, about $40 \%$ of the total emission of the inner disk comes from discrete sources while this contribution drops to $20 \%$ in the outer disk. The dust temperature, estimated from the 70 and $160 \mu \mathrm{m}$ emission, decreases from 25 to $21 \mathrm{~K}$ from the center to $4 \mathrm{kpc}$ and, after a plateau, it might increase at the edge of the star-forming disk. The average ratio between the TIR and FIR luminosities is $1.40 \pm 0.04$ across the whole disk.

- We examine the SFR of M33 from various tracers (extinction corrected $\mathrm{H} \alpha$ and FUV, TIR, FIR, $24 \mu \mathrm{m}$ ). We estimate the total integrated SFR to be $0.45 \pm 0.1 M_{\odot} \mathrm{yr}^{-1}$. From the $\mathrm{H} \alpha$ luminosity (which accounts for SF in the last $<10 \mathrm{Myr}$ ) we infer a lower value, $\operatorname{SFR}(\mathrm{H} \alpha)=0.35 M_{\odot} \mathrm{yr}^{-1}$, while the FUV emission (which accounts for SF in the last $<100 \mathrm{Myr}$ ) gives $\operatorname{SFR}(\mathrm{FUV})=0.55 M_{\odot} \mathrm{yr}^{-1}$; the difference is small with respect to the uncertainties, so that we cannot conclude definitively that the SFR has been declining in the last 100 Myr. If we calibrate the SFR from FIR and TIR emission through the values from UV data, we obtain conversion factors in the upper range of what is generally found for latetype galaxies, likely due to the low dust content of M33.

- A SFR diagnostic based on the $24 \mu \mathrm{m}$ emission in M 33 gives a conversion factor more than three times higher than the standard ones. To estimate the validity of the $24 \mu \mathrm{m}$ emission as a SFR tracer away from H II complexes, we investigated the nature of its high diffuse fraction. We find that VSG emission, as powered by the diffuse ISRF, accounts for only $10 \%$ of the diffuse $24 \mu \mathrm{m}$ emission, while unresolved AGB stars of intermediate mass, carbon stars, through their dusty circumstellar envelopes, can provide most of the diffuse $24 \mu \mathrm{m}$ emission. If indeed evolved stars contribute substantially to the $24 \mu \mathrm{m}$ brightness, emission at $24 \mu \mathrm{m}$ can trace the SFR about 1 Gyr ago. In this paper we only give a lower limit to the SFR of about $0.20 M_{\odot} \mathrm{yr}^{-1}$. This estimate takes into account only the diffuse $24 \mu \mathrm{m}$ emission and does not consider the most luminous CSs, which might be resolved as discrete sources, as well as the fraction of CSs without dusty shells.
Table A.1. Midplane to emergent intensity ratio $J_{v}(0) / I(0, \mu)$.

\begin{tabular}{l|ccc}
\hline \hline$\tau_{0} / \mu$ & $\beta=\frac{1}{1}$ & $\beta=\frac{1}{2}$ & $\beta=\frac{1}{3}$ \\
\hline 0.1 & 0.53 & 0.52 & 0.51 \\
0.6 & 0.65 & 0.56 & 0.53 \\
1.6 & 0.83 & 0.54 & 0.42 \\
3.6 & 0.97 & 0.36 & 0.22 \\
6.6 & 1.00 & 0.24 & 0.12 \\
\hline
\end{tabular}

With the help of future surveys of evolved stars in M 33, it should soon be possible to complement our study and obtain a better view of the time evolution of the SFR in the Triangulum galaxy.

Acknowledgements. We would like to thank Rene Walterbos for providing us the $\mathrm{H} \alpha$ image of M33, David Thilker for the GALEX-UV profiles and George Helou for discussions and advice. We also thank the anonymous referee whose insightful comments improved the clarity of the paper. The work of S. V. is supported by a INAF-Osservatorio Astrofisico di Arcetri fellowship. The Spitzer Space Telescope is operated by the Jet Propulsion Laboratory, California Institute of Technology, under contract with the National Aeronautics and Space Administration. This research has made use of the NASA/IPAC Extragalactic Database, which is operated by JPL/Caltech, under contract with NASA.

\section{Appendix A: A simplified exponential atmosphere}

We model the disk as a plane parallel medium symmetric with respect to midplane. In the case of isotropic extinction and emissivity, at vertical distance $z$ from midplane, the transfer of the intensity $I_{v}(z, \theta)$ along a direction at angle $\theta$ from the vertical will follow

$\mu \frac{\mathrm{d} I_{v}(z, \theta)}{\chi_{v}(z) \mathrm{d} z}=S_{v}(z)-I_{v}(z, \theta)$,

as usual $\mu=\cos \theta, \chi_{v}$ is the volume extinction coefficient, and $S_{v}$ is the source function; $S_{v}(z)=\epsilon_{v}(z) / \chi_{v}(z)$, where $\epsilon_{v}(z)$ is the volume emissivity. If $\tau_{v}$ is the vertical optical thickness $\tau_{v}(z)=$ $\int_{z}^{\infty} \chi_{v}\left(z^{\prime}\right) d z^{\prime}$, then

$\mu \frac{\mathrm{d} I_{v}(\tau, \mu)}{\mathrm{d} \tau}=I_{v}(\tau, \mu)-S_{v}(\tau)$

If we assume, though not strictly required, that the intensity at the midplane is also isotropic, $I_{v}(z=0, \theta)=J_{v}(0)$, then the intensity emerging from the atmosphere $(z=+\infty, \tau=0, \mu>0)$ will be

$I_{\nu}(0, \mu)=J_{v}(0) \mathrm{e}^{-\tau_{0} / \mu}+\int_{0}^{\tau_{0}} S(\tau) \mathrm{e}^{-\tau / \mu} \frac{\mathrm{d} \tau}{\mu}$,

where $\tau_{0}=\tau(z=0)$ is the optical half-thickness of the disk. At the same time, at midplane $\left(z=0, \tau=\tau_{0}, \mu<0\right)$, if there is no incoming radiation from outside the disk, we have

$I_{v}\left(\tau_{0}, \mu\right)=J_{v}(0)=\int_{0}^{\tau_{0}} S(\tau) \mathrm{e}^{-\left(\tau_{0}-\tau\right) / \mu} \frac{\mathrm{d} \tau}{\mu}$

If the extinction and emissivity volume coefficients depend exponentially on $z$, so will be $S_{v}(z)$, and the integrals in Eqs. (A.3), (A.4) can be evaluated in terms of Incomplete Gamma functions $\gamma\left(\beta, \pm \tau_{0} / \mu\right)$, where $\beta$ is the ratio between the extinction and emissivity scale heights, $\beta=h_{\mathrm{ext}} / h_{\mathrm{em}}$. By 
comparing the two solutions we find the emergent intensity $I_{v}(\tau=0, \mu)$ in terms of the one at midplane $J_{v}(0)$, and vice versa:

$I_{\nu}(0, \mu)=J_{v}(0) \mathrm{e}^{-\tau_{0} / \mu}+J_{v}(0) \frac{\mathrm{e}^{\tau_{0} / \mu} \gamma\left(\beta, \tau_{0} / \mu\right)}{(-1)^{\beta} \gamma\left(\beta,-\tau_{0} / \mu\right)}$.

Some numerical values of $J_{v}(0) / I(0, \mu)$, the ratio between midplane and emergent intensity, are shown in Table A.1. For $\beta=1$ we recover the values for the particular case of constant $S_{v}$.

\section{References}

Bendo, G. J., Draine, B. T., Engelbracht, C. W., et al. 2008, ArXiv e-prints, 806 Bertin, E., \& Arnouts, S. 1996, A\&AS, 117, 393

Bianchi, L., Thilker, D. A., Burgarella, D., et al. 2005, ApJ, 619, L71

Buat, V., \& Xu, C. 1996, A\&A, 306, 61

Calzetti, D. 2001, PASP, 113, 1449

Calzetti, D., Kennicutt, Jr., R. C., Bianchi, L., et al. 2005, ApJ, 633, 871

Cannon, J. M., Walter, F., Armus, L., et al. 2006, ApJ, 652, 1170

Cioni, M. L., Irwin, M., Ferguson, A. M. N., et al. 2008, ArXiv e-prints, 805

Corbelli, E. 2003, MNRAS, 342, 199

Corbelli, E., \& Salucci, P. 2000, MNRAS, 311, 441

Corbelli, E., \& Schneider, S. E. 1997, ApJ, 479, 244

Corbelli, E., \& Walterbos, R. A. M. 2007, ApJ, 669, 315

Dale, D. A., \& Helou, G. 2002, ApJ, 576, 159

Dale, D. A., Bendo, G. J., Engelbracht, C. W., et al. 2005, ApJ, 633, 857

Deul, E. R., \& van der Hulst, J. M. 1987, A\&AS, 67, 509

Devereux, N., Duric, N., \& Scowen, P. A. 1997, AJ, 113, 236

Draine, B. T., \& Lee, H. M. 1984, ApJ, 285, 89

Draine, B. T., \& Li, A. 2007, ApJ, 657, 810

Elmegreen, B. G., \& Hunter, D. A. 2006, ApJ, 636, 712

Engargiola, G., Plambeck, R. L., Rosolowsky, E., \& Blitz, L. 2003, ApJS, 149, 343

Engelbracht, C. W., Blaylock, M., Su, K. Y. L., et al. 2007, PASP, 119, 994 Engelbracht, C. W., Gordon, K. D., Rieke, G. H., et al. 2005, ApJ, 628, L29 Fazio, G. G., Hora, J. L., Allen, L. E., et al. 2004, ApJS, 154, 10

Freedman, W. L., Wilson, C. D., \& Madore, B. F. 1991, ApJ, 372, 455

Gil de Paz, A., Boissier, S., Madore, B. F., et al. 2007, ApJS, 173, 185

Girardi, L., Bressan, A., Bertelli, G., \& Chiosi, C. 2000, A\&AS, 141, 371

Greenawalt, B. E. 1998, Ph.D. thesis, New Mexico State University

Groenewegen, M. A. T., Wood, P. R., Sloan, G. C., et al. 2007, MNRAS, 376, 313

Haas, M., Klaas, U., \& Bianchi, S. 2002, A\&A, 385, L23

Herwig, F. 2005, ARA\&A, 43, 435

Heyer, M. H., Corbelli, E., Schneider, S. E., \& Young, J. S. 2004, ApJ, 602, 723

Hippelein, H., Haas, M., Tuffs, R. J., et al. 2003, A\&A, 407, 137

Hirashita, H., Hibi, Y., \& Shibai, H. 2007, MNRAS, 379, 974
Hoopes, C. G., \& Walterbos, R. A. M. 2000, ApJ, 541, 597

Issa, M. R., MacLaren, I., \& Wolfendale, A. W. 1990, A\&A, 236, 237

Jarrett, T. H., Chester, T., Cutri, R., Schneider, S. E., \& Huchra, J. P. 2003, AJ, 125,525

Kennicutt, Jr., R. C. 1989, ApJ, 344, 685

Kennicutt, Jr., R. C. 1998, ARA\&A, 36, 189

Kennicutt, Jr., R. C., Calzetti, D., Walter, F., et al. 2007, ApJ, 671, 333

Kroupa, P. 2001, MNRAS, 322, 231

Kruegel, E. 2003, The physics of interstellar dust, ed. E. Kruegel, IoP Series in astronomy and astrophysics, Bristol, UK, The Institute of Physics

Laor, A., \& Draine, B. T. 1993, ApJ, 402, 441

Leitherer, C., Schaerer, D., Goldader, J. D., et al. 1999, ApJS, 123, 3

Magrini, L., Corbelli, E., \& Galli, D. 2007a, A\&A, 470, 843

Magrini, L., Vílchez, J. M., Mampaso, A., Corradi, R. L. M., \& Leisy, P. 2007b, A\&A, 470, 865

Makovoz, D., \& Marleau, F. R. 2005, PASP, 117, 1113

Martin, D. C., Fanson, J., Schiminovich, D., et al. 2005, ApJ, 619, L1

Massey, P., Armandroff, T. E., Pyke, R., Patel, K., \& Wilson, C. D. 1995, AJ, 110,2715

Mathis, J. S., Rumpl, W., \& Nordsieck, K. H. 1977, ApJ, 217, 425

McConnachie, A. W., Chapman, S. C., Ibata, R. A., et al. 2006, ApJ, 647, L25

McQuinn, K. B. W., Woodward, C. E., Willner, S. P., et al. 2007, ApJ, 664, 850

Miyake, K., \& Nakagawa, Y. 1993, Icarus, 106, 20

O'Halloran, B., Satyapal, S., \& Dudik, R. P. 2006, ApJ, 641, 795

Pérez-González, P. G., Kennicutt, Jr., R. C., Gordon, K. D., et al. 2006, ApJ, 648, 987

Reach, W. T., Megeath, S. T., Cohen, M., et al. 2005, PASP, 117, 978

Regan, M. W., \& Vogel, S. N. 1994, ApJ, 434, 536

Rice, W., Boulanger, F., Viallefond, F., Soifer, B. T., \& Freedman, W. L. 1990, ApJ, 358, 418

Rieke, G. H., Young, E. T., Engelbracht, C. W., et al. 2004, ApJS, 154, 25

Rosolowsky, E., \& Simon, J. D. 2008, ApJ, 675, 1213

Rowe, J. F., Richer, H. B., Brewer, J. P., \& Crabtree, D. R. 2005, AJ, 129, 729

Salpeter, E. E. 1955, ApJ, 121, 161

Seibert, M., Martin, D. C., Heckman, T. M., et al. 2005, ApJ, 619, L55

Tabatabaei, F. S., Beck, R., Krügel, E., et al. 2007a, A\&A, 475, 133

Tabatabaei, F. S., Krause, M., \& Beck, R. 2007b, A\&A, 472, 785

Thilker, D. A., Bianchi, L., Meurer, G., et al. 2007a, ApJS, 173, 538

Thilker, D. A., Boissier, S., Bianchi, L., et al. 2007b, ApJS, 173, 572

Thilker, D. A., Hoopes, C. G., Bianchi, L., et al. 2005, ApJ, 619, L67

Verley, S., Hunt, L. K., Corbelli, E., \& Giovanardi, C. 2007, A\&A, 476, 1161

Weingartner, J. C., \& Draine, B. T. 2001, ApJS, 134, 263

Werner, M. W., Roellig, T. L., Low, F. J., et al. 2004, ApJS, 154, 1

Whitcomb, S. E., Gatley, I., Hildebrand, R. H., et al. 1981, ApJ, 246, 416

Whittet, D. C. B. 1992, Dust in the galactic environment (Institute of Physics Publishing), 306

Wu, H., Cao, C., Hao, C.-N., et al. 2005, ApJ, 632, L79

Yun, M. S., Reddy, N. A., \& Condon, J. J. 2001, ApJ, 554, 803

Zubko, V. G., Mennella, V., Colangeli, L., \& Bussoletti, E. 1996, MNRAS, 282, 1321 